\title{
Goal Recognition for Deceptive Human Agents through Planning and Gaze
}

Thao Le

Ronal Singh

Tim Miller

School of Computing and Information Systems

The University of Melbourne, Parkville VIC 3010, Australia
THAOL4@STUDENT.UNIMELB.EDU.AU

RR.SINGH@UNIMELB.EDU.AU

TMILLER@UNIMELB.EDU.AU

\begin{abstract}
Eye gaze has the potential to provide insight into the minds of individuals, and this idea has been used in prior research to improve human goal recognition by combining human's actions and gaze. However, most existing research assumes that people are rational and honest. In adversarial scenarios, people may deliberately alter their actions and gaze, which presents a challenge to goal recognition systems. In this paper, we present new models for goal recognition under deception using a combination of gaze behaviour and observed movements of the agent. These models aim to detect when a person is deceiving by analysing their gaze patterns and use this information to adjust the goal recognition. We evaluated our models in two human-subject studies: (1) using data collected from 30 individuals playing a navigation game inspired by an existing deception study and (2) using data collected from 40 individuals playing a competitive game (Ticket To Ride). We found that one of our models (Modulated Deception Gaze+Ontic) offers promising results compared to the previous state-of-the-art model in both studies. Our work complements existing adversarial goal recognition systems by equipping these systems with the ability to tackle ambiguous gaze behaviours.
\end{abstract}

\section{Introduction}

The problem of goal recognition involves inferring an agent's goal by observing their actions. There are numerous methods identified by Sukthankar et al. (2014), and among these is the model-based approach. There are at least two conventional approaches to model-based goal recognition. The first approach uses the ontic actions (physical actions that change the state of the world) of agents to estimate the probabilities of candidate goals (Ramírez \& Geffner, 2010; Masters \& Sardina, 2017a; Pereira et al., 2017).

More recently, Singh et al. $(2018,2020)$ have exploited the fact that visual behaviour is intrinsically linked to the way that people plan and execute actions. In short: the gaze of our eyes gives information to observers about our future actions and people use this to determine our actions, even when we are trying to be deceptive. Singh et al. (2018, 2020) define a goal recognition model that incorporates the gaze behaviour of human agents to infer goals. However, this model assumes that individuals are rational and honest; for example, people may minimise action costs when achieving their goals. As such, these works do not consider people deliberately altering their actions.

In deceptive or adversarial scenarios, people may intentionally exhibit ambiguous actions, physical or gaze, posing a clear challenge for goal recognition systems that assume 


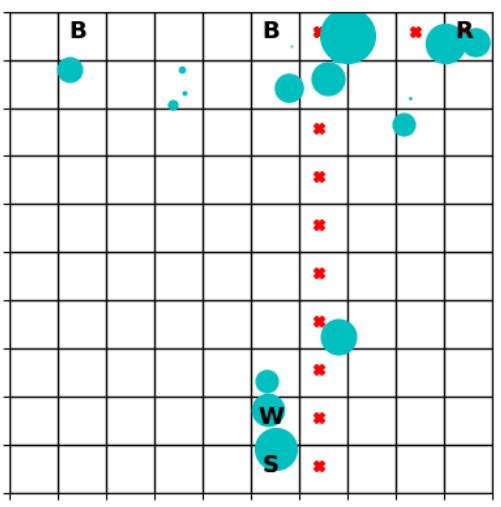

(a) Example of Natural Gaze

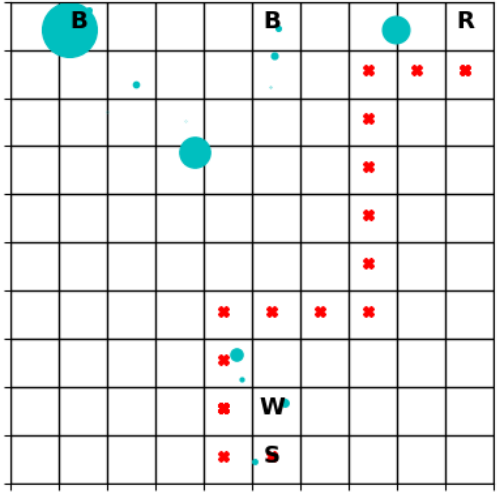

(b) Example of Deceptive Gaze

Figure 1: An example of the natural gaze and deceptive gaze in the first study. Blue circles are the fixation points (the points on which the eyes are focused for at least $200 \mathrm{~ms}$ ). Bigger circle implies a higher fixation duration. Red $\mathbf{x}$ represents the agent's ontic actions. Notations of the maps: $\mathbf{S}$ is the starting point, $\mathbf{B}$ is the bogus goal, $\mathbf{R}$ is the real goal and $\mathbf{W}$ is the wall.

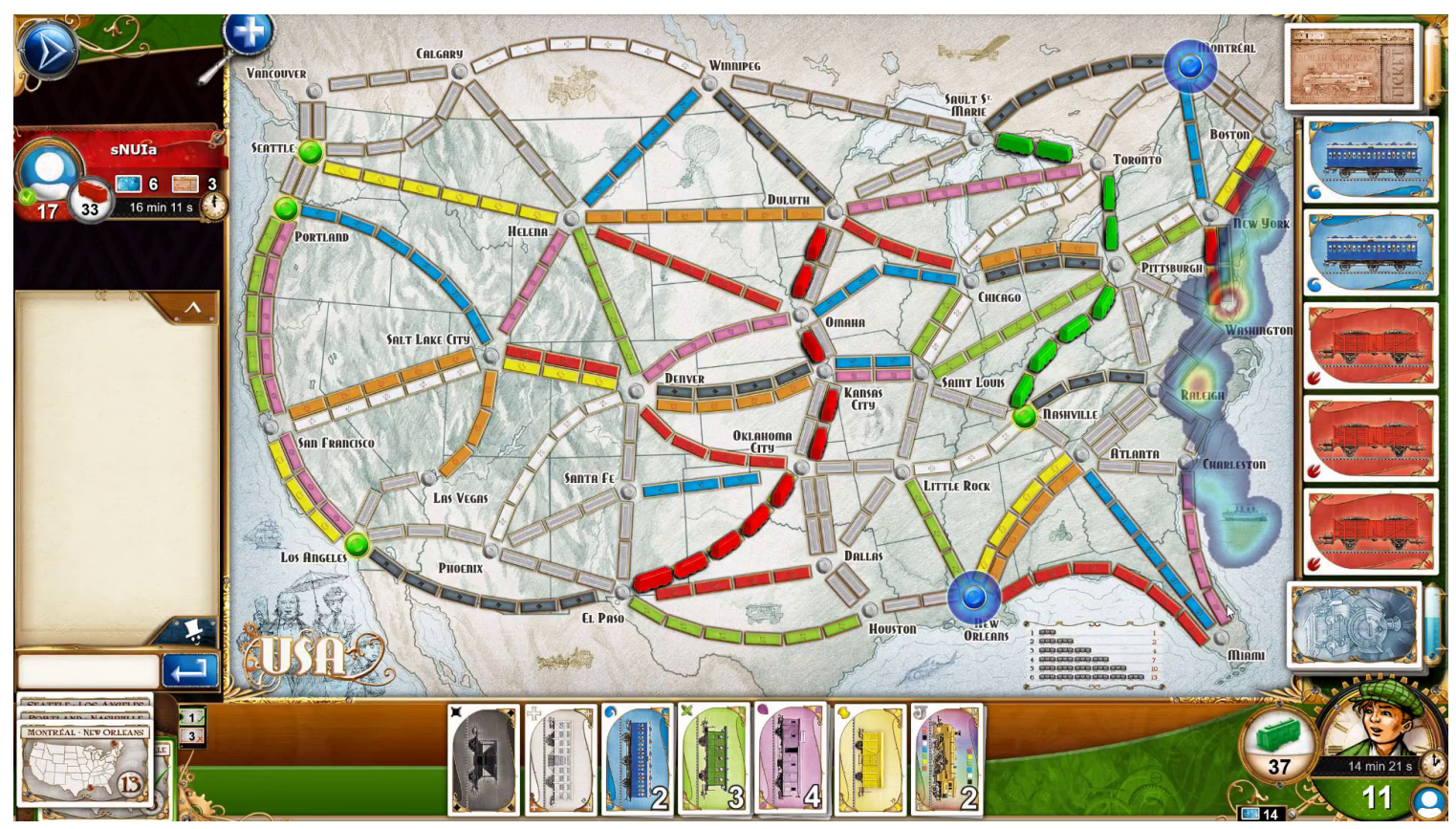

Figure 2: An example of gaze visualization in the second study (Ticket To Ride game). This figure is taken from Newn et al. (2017). The gaze visualization is displayed between Boston and Miami (on the right of the board). 
rationality. An example from our first study is in Figure 1, which shows a participant's deceptive and natural gaze as they navigate a vehicle from cell $\mathbf{S}$ to cell $\mathbf{R}$. In these figures, the circles represent where a person's gaze was fixated. A larger circle means the duration of the fixation was longer. In Figure 1a, the gaze naturally follows the ontic actions (red) and the fixations are higher for the real goal (R). But when the participant was instructed to be deceptive with their gaze in Figure 1b, fixation points are located far from the ontic actions and the participant mostly fixated for a longer period at one of the bogus goals (B). Figure 2 shows an example of how gaze is presented in the second study, which was based on a multiplayer board game called Ticket to Ride (discussed in detail in Section 4.2). In this game, players have to claim train routes to connect cities. In the example instance of this game shown in Figure 2, the gaze (heatmap) shows that the player might have an intention to claim any routes between Boston and Miami. We can combine the information derived from the gaze data with information about completed in-game actions, for example, the red player has already claimed a route between cities Boston and Washington, to predict players intentions. In this case, probably the player is interested in claiming routes between Boston and Miami.

Masters and Sardina (2019) tackle deceptive ontic actions by quantifying the degree of rationality of a sequence of actions using a rationality measure (RM). The basis of the model is that if a sequence of actions is far from optimal for any candidate goal, the agent is behaving irrationally. Their rationality measure is used to moderate the confidence of a prediction. As far as we are aware, there is no work along the lines of Masters and Sardina (2017b) for deceptive gaze behaviours. Earlier work, such as shown by Newn et al. (2018) that identified various deceptive gaze strategies in digital board games do not propose how to infer goals algorithmically. However, they argued that the players could not prevent leaking information through their gaze, highlighting the prospects of utilising gaze for goal recognition even in the presence of ambiguous gaze. While there are some works on adversarial and exploratory goal recognition (Gal et al., 2012; Geib \& Goldman, 2001), none of them incorporated gaze.

In this paper, we propose a goal recognition model that combines model-based (onticbased) goal recognition with gaze-based goal recognition to infer goals of people acting deceptively. This model extends an existing model for gaze-based goal recognition (Singh et al., 2018, 2020) by identifying and formalising two key measures: (1) honesty degree; and (2) visual acuity. The honesty degree measure aims to detect and categorise potentially deceptive gaze behaviours and quantify deception when we do not know the goal of a person. Like Masters and Sardina (2019)'s rationality measure, we use the honesty degree to moderate the prediction confidence by modulating the likelihood of the candidate goals. The purpose of modulating the probability distribution is to increase the probability of a true goal, and at the same time lower the probabilities of deceptive goals. The visual acuity measure considers the non-central foveal vision (Duchowski, 2018); that is, the field of vision outside of the central areas. We use these factors to devise gaze models to tackle deceptive gaze behaviours, and evaluate the performance of these models individually and in combination with existing models, such as those by Singh et al. (2018) and Singh et al. (2020), by replacing their gaze model with gaze models that explicitly consider deception. Singh et al. (2018) combined gaze and ontic actions to predict goals more accurately and 
earlier. An ontic action is an action that changes the state of the world. Gaze is not an ontic action since it does not change the state of the world.

The key contribution of this work is a goal recognition model and variations designed that combines both gaze actions and ontic actions under deceptive gaze behaviours. We build our model by extending the earlier work from Masters and Sardina (2017b), Singh et al. $(2018,2020)$ to take into account the deceptive actions. Even though gaze is a noisy signal, we can infer useful information under deception, including when the gaze itself is deceitful. Further, we have defined new deceptive models, such as: Deception Gaze (DG), Modulated Deception Gaze (MDG), Deception Gaze + Ontic (DGO) and Modulated Deception Gaze+Ontic (MDGO), which have never been defined in the previous work (Singh et al., 2018, 2020). These models are defined based on two new measures honesty degree and visual acuity. We evaluated the models on two different studies while the participants' gaze data and game actions were being recorded. The first game is a single-player VIP routing game, in which participants must navigate a vehicle containing a VIP to a destination. The second game is a multi-player game Ticket To Ride that was used in previous works (Newn et al., 2017, 2018; Singh et al., 2018). We compared our models against a recent gaze-based goal recognition model (Singh et al., 2018, 2020). Although the model proposed in Singh et al. $(2018,2020)$ is reasonably accurate goal recognition model that incorporates gaze, our results showed that our enhanced gaze-model more accurately predicted the goals of players in deceptive scenarios $(p<0.05)$, while remaining robust in non-deceptive scenarios. These contributions are significant because they advance the capabilities of existing gaze-based predictive systems at tackling uncertainty in gaze, and the extreme case, deceptive gaze behaviours.

This paper is organised as follows. In Section 2, we discuss the relevant background of model-based goal recognition, gaze recognition, deceptive gaze strategies and detecting deception. In Section 3, we present the proposed models to handle deceptive gaze behaviours by explaining honesty degree and visual acuity measures. Section 4 describes the design of two human-subject experiments: the first study is a simple single-player navigational game and the second study is a more complex multi-player board game (Ticket To Ride). In

Section 5, we explain the results of our proposed models compared to previous state-of-theart models. Section 6 presents our discussion on the results.

\section{Background and Related Work}

In this section, we review related works on goal and intentions recognition, such as modelbased and gaze-based approaches, and deceptive gaze behaviours.

\subsection{Model-Based Goal Recognition}

In a Goal recognition $(G R)$ problem, we aim to find an agent's (human or artificial) goal by observing their behaviour. Goal recognition can be classified into three categories (Carberry, 2001): keyhole recognition, in which an agent does not intend to change its behaviour; intended recognition, whereby the agent attempts to reveal its real goal; and adversarial recognition when an agent hides its real goal. There are many keyhole goal recognition algorithms for honest, natural and rational behaviours (Ramírez \& Geffner, 2009; Masters 
\& Sardina, 2017a; Vered, Kaminka, \& Biham, 2016; Singh et al., 2018), as well as for adversarial settings, such as (Keren, Gal, \& Karpas, 2015; Masters \& Sardina, 2019).

The basic approach to model-based goal recognition is to observe a sequence of actions performed by an agent, and for each of the candidate goals, identify whether this behaviour is rational. In these models, 'rational' typically means that the observed behaviour is low cost or high quality for a given goal. The relative rationality of the sequence relative to goals is used to derive a probability distribution over the goals, estimating the likelihood that each candidate goal is the real goal.

Most of these models assume that an agent is behaving honestly or rationally. However, Masters and Sardina (2019) propose the rationality measure (RM) to quantify the degree of rationality, and apply this measure to the posterior probabilities of the goals to self-regulate and lower confidence in the predictions when the irrational behaviour is detected. When the behaviour becomes more irrational, the probabilities are distributed more uniformly. This model can be applied when agents are deceptive.

The variable $\beta$ is what is novel about the model. This refers to the rationality measure, defined as follows:

$$
\beta=\left(\max _{g \in G} \frac{\operatorname{optc}(s, g)}{\operatorname{optc}(s, g, O)}\right)^{\gamma}
$$

where $G$ is the set of all goals and $\gamma$ regulates how much the certainty should decrease when the irrational behaviour is detected; $s$ and $g$ are the start and goal locations, respectively, $O$ is the sequence of observations, $\operatorname{optc}(s, g, O)$ is the sum of the cumulative cost from the start location to the current location and the optimal cost from the current location to the goal, optc $(s, g)$ is the optimal cost of solution path from a starting point $s$ to a goal $g$.

The likelihood of a goal is defined as:

$$
P(O \mid g)=\frac{1}{e^{\beta \cdot \operatorname{costdiff}(s, g, O)}}
$$

where costdiff $(s, g, O)=\operatorname{optc}(s, O, g)-\operatorname{optc}(s, g)$ is the difference between the optimal and observed costs.

And the posterior probability of a goal is:

$$
P(g \mid O)=\kappa P(O \mid g)
$$

where $\kappa$ is the normalising constant that normalises the probability of each goal relative to the others. Thus, the probability assigned to each goal is proportional to how much it explains the observed behaviour.

Thus, $\beta=1$ if the observed sequence $O$ is optimal for at least one goal, however $\beta<1$ if the sequence $O$ is non-optimal for all goals, and becomes lower the less optimal it is. The influence of $\beta$ on Equation 3 is to modulate the goal recognition, pushing down the probability of likely goals relative to less likely goals, because the agent is deemed more irrational, therefore, their behaviour is harder to predict.

In the event that $\beta=1$, Equation 3 is a goal recognition model that assumes rationality, which is called Ontic (O) model. Moreover, we assign $\gamma=1$ and then calculate $\beta$ based on $\gamma$ as in Equation 1 to have Modulated Ontic (MO) model. 


\subsection{Gaze and Intention Recognition}

Human vision has different visual acuity that depends on the visual angle. High visual acuity is limited to the central of $2^{\circ}$, which is called the foveal vision. Outside the foveal vision, the vision area is classified into parafoveal vision $\left(5^{\circ}\right)$, perifoveal vision $\left(10^{\circ}\right)$ and peripheral vision $\left(80^{\circ}\right)$ (Duchowski, 2018). Due to the limited angle of foveal vision $\left(2^{\circ}\right)$, our eyes constantly move to reposition the fovea. These movements result in various types of eye movements, such as fixations and saccades (Qvarfordt, 2017; Duchowski, 2007, 2018). During a fixation, gaze maintains at a single position. Saccades are eye movements from one point to another point. The non-foveal vision and deceptive fixation points are common in sports, for example, martial arts (Hausegger, Vater, \& Hossner, 2019). As such, we believe that intention recognition model needs to be able to leverage an understanding of different types of eye movements and non-foveal vision to tackle ambiguous gaze behaviours.

The idea that visual behaviour is linked to how humans plan and execute actions (Land, 2009) has inspired several computational models for gaze-based intention recognition, both machine learning based (Bednarik et al., 2013; Huang et al., 2015; Koochaki \& Najafizadeh, 2019) and model based (Admoni \& Srinivasa, 2016; Singh et al., 2018).

Singh et al. (2018) propose a model that combines gaze input with a model-based goal recognition approach for intention and goal recognition. Formally, for each intention $i$, they record the accumulated fixation count and fixation duration. The standard fixation duration is 200ms (Singh et al., 2020), count $_{i}$ and natural_duration ${ }_{i}$ respectively where

natural_duration $_{i}=\sum_{j=1}^{\text {count }_{i}}$ duration $_{i, j}$. Here count $_{i}$ represents the number of times the intention $i$ was signalled and duration $_{i, j}$ represents the fixation duration of gaze point $j$ at intention $i$, where $1 \leq j \leq$ count $_{i}$. Using these two gaze features, they compute the fixation score of an intention $i$ as follows:

$$
s_{i}=\log \left(\lambda \cdot \text { natural_duration }_{i}+(1-\lambda) \cdot \text { count }_{i}\right)
$$

The parameter $\lambda \in[0,1]$ is the relative weight between fixation duration and fixation count. To get the probability of each goal, $i$, the fixation scores are normalised using the sum of fixation score of all intentions. We call this the Natural Gaze (G) model. Formally:

$$
P\left(i \mid O_{g}\right)=\frac{s_{i}}{\sum_{j \in I} s_{j}}
$$

where $I$ is a set of possible intentions (goals); $O_{g}$ is a set of gaze observations, in which includes a set of fixation points. A fixation point is measured based on fixation duration and count. Finally, they compute the posterior probability of each intention based on both gaze and physical actions as follows:

$$
P\left(i \mid O_{a}, O_{g}\right)=P\left(i \mid O_{g}\right) \cdot P\left(O_{a} \mid i\right)
$$

where $P\left(O_{a} \mid i\right)$ is a default model-based goal recognition model, such as Ramírez and Geffner (2010). Evaluations of their model show that incorporating gaze increases predicted accuracy, enables correct predictions to be made earlier, and has no additional computational cost in the game Ticket to Ride (Singh et al., 2018). We call this the Natural Gaze+Ontic (GO) model.

However, Singh et al. (2018) assume that the gaze action of the player is truthful. In this paper, we build on this model to take into account the deceitful gaze. 


\subsection{Deceptive Gaze Strategies}

In a general theory of deception, Whaley (1982) and Bell (2003) define simulation and dissimulation as two basic strategies. Dissimulation is obscuring the truth, such as hiding our real goal from an observer; whereas simulation is displaying the false, such as trying to make an observer believe a bogus goal is our real goal. Based on these two foundations, we could define the strategies of deceptive behaviours.

Many gaze features have been explored to detect deception. For example, pupil dilation is higher for deceivers than truthful people (Proudfoot et al., 2016). Similarly, in penalty shootouts, players had extended fixation duration on the side to which they did not shoot, fooling the goalkeeper (Wood et al., 2017). Student cheating in exams often uses peripheral vision (Aravena et al., 2017). These works demonstrate the promise of used gaze as a means to detect and quantify gaze-based deception.

Newn et al. (2018) investigate deceptive gaze behaviours in an online competitive gameplay environment. They identified and classified gaze-based deception behaviours into eight distinct strategies: Gaze Averting, Gaze Scattering, Information Reduction, Risk Reduction, Obstruction, Gaze Misdirection, Decoys and Gaze Camouflage. For example, Gaze Averting is one of the dissimulation strategies in which players try to avoid looking at their intended goals in the game. A key result of this work was that even players who knew their gaze was being tracked and displayed to their adversary could not prevent leaking information through their gaze, making gaze a useful indicator of human goals, even though they are deceiving. However, they did not build a computational model of this.

\subsection{Detecting Deception}

Detecting deception is a challenging problem. Although the eye gaze is useful to infer others' intentions, we can make false judgements when observing behavioural cues. For example, people tend to believe that avoiding eye contact is a sign of deceit, but gaze aversion can be a signal of lacking confidence or cultural norms. Therefore, Wheeler (2004) indicated that we should avoid this mistake when concluding a person is whether truthful or deceitful. Moreover, it is hard to distinguish between deceptive and honest gaze, as deceivers often have a desire to persuade people by maintaining their eye contact. To solve this problem, Mann et al. (2012) classified two types of eye contact: traditional eye contact and deliberate eye contact. The authors define that deliberate eye contact happens when someone looks into the eyes in a longer amount of time than it would be normally expected. By considering the deliberate eye contact, they found that deceptive people imposed more deliberate eye gaze than honest ones. This finding is similar to the Gaze Misdirection strategy as defined by Newn et al. (2018).

Although most of the studies have used multimodal features as the effective inputs to detect deception, Bhaskaran et al. (2011) only used eye movements as the input and could predict deception with $82.5 \%$ accuracy. They conducted a study to extract the normal behaviours and deceptive behaviours of the subjects in an interrogation. A dynamic Bayesian model was employed to differentiate between deceptive and non-deceptive behaviours. This study shows that using multimodal features might be unnecessary as some features are not correlated with the deceptive behaviour. 
Typically, machine learning is used to solve the detecting deception problems. Zhou and Zhang (2012) showed that verbal and nonverbal cues can be used in the classification task to detect deception. Although this study focused on extracting linguistic features, it suggested that machine learning can be used to recognise deception based on nonverbal behaviour. Demyanov et al. (2015) employed logistic regression to classify between truthful and deceptive people based on the movements of eyebrows, eyes and mouth. This research also contributed a large Mafia database for future work. Pérez-Rosas et al. (2015) conducted a study to differentiate between honest and dishonest statements of the testimonies from real-life trials. They used both verbal and non-verbal features; then applied Decision Trees and Random Forest approaches to build the deception classifiers. A drawback of this approach is that it requires sufficient training data, which may not always be available. In this paper, we design new models based on scientific studies, which allows use in new situations without obtaining new data sets.

\section{Gaze Model for Deceptive Gaze Behaviours}

In this section, we first introduce two key measures: Honesty Degree and Visual Acuity. The main purposes of the Honesty Degree are to modulate the probability distribution of all intentions and allow us to detect and quantify gaze-based deceptive behaviours. The Visual Acuity measure is proposed to increase the fixation score of the intentions outside the foveal vision and therefore increase the probability of the real goal. Using these, we extend earlier work from Masters and Sardina (2017b), Singh et al. $(2018,2020)$ to obtain more accurate goal recognition. Our gaze models enhance the previous models in terms of the ability of the models to handle deceptive gaze behaviours.

\subsection{Problem Formulation}

We formulate the problem of goal recognition based on existing definitions (Vered et al., 2016; Singh et al., 2018, 2020). We have a set of possible goals, a set of actions that achieve the goals, and a sequence of actions that form a partial plan to one of the goals. The goal recognition problem is to determine the likelihood of the different goals given the observations. In our problem, we receive two types of observations: (1) ontic actions; and (2) gaze actions. Ontic actions modify the 'physical' world, while gaze observations tell us which 'regions' of the world the human is looking at. For example, in an online game, gaze actions are those that look at the different areas of the game that a person is looking at, such as pieces and game elements. Thus, we consider fixations on regions as the possible

gaze actions $G$ on those regions defined in the context of the online goal recognition problem as follows.

Formally, Singh et al. $(2018,2020)$ define an online goal recognition problem $R$ as a tuple $R=\left\langle W, s_{0}, I, A, G, O_{a}, O_{g}\right\rangle$. W is the world in which the agent operates, $s_{0} \in W$ is the initial state of the observations, $I$ is the possible set of intentions (goals), $A$ is the set of ontic actions available to achieve the intentions, $G$ is the set of possible gaze actions, $O_{a}$ is the sequence of ontic action observations (made up of actions from $A$ ), and $O_{g}$ is the set of gaze observations (made up of observations from $G$ ). The problem is to derive a probability distribution over $I$ that estimates the likelihood that each intention is the agent's potential intention (relative to each other) based on the observed sequence $O_{a}$ and $O_{g}$. 
The solution for this problem proposed by Singh et al. $(2018,2020)$ assumes that $O_{g}$ is honest, that is, the gaze observations are natural without ambiguity or deception. However, our model relaxes this assumption and incorporates the Honesty Degree and Visual Acuity to handle deceptive gaze behaviours.

\subsection{Honesty Degree}

We propose the honesty degree of a single intention with two main purposes: 1) to detect deception; and 2) to use as a parameter to modulate the certainty over the probability distribution of candidate intentions. The modulation will assist in moderating the confidence of the predictions, similar to Masters and Sardina (2019).

The average fixation duration of a human is around 300 milliseconds (Qvarfordt, 2017). Therefore, we consider high values to be an indicator of deceptive behaviour; that is, the higher the average fixation duration, the higher the chance of deception. There are many examples in sports for this, such as martial arts (Hausegger et al., 2019), where fighters have been known to use longer fixations on bogus goals and peripheral vision. Based on this idea, we provide a definition of honesty degree $h$ (natural_duration $_{i}$ ) of an intention $i$ in the gaze-based context as follows:

$$
h\left(\text { natural_duration }_{i}\right)=\frac{1}{1+e^{\alpha\left(\text { natural_duration }_{i}-\text { DurationThreshold }\right)}}
$$

where natural_duration $_{i}$ is the fixation duration value (ms) of intention $i$, which is defined as natural_duration $i=\sum_{j=1}^{\text {count }_{i}}$ duration $_{i, j}$ (based on Equation 4). DurationThreshold is the fixation duration threshold such that at the threshold point, the honesty degree is 0.5 ; and $\alpha$ is the rate parameter. In our experiments, we chose DurationThreshold $=350$ (ms) based on prior work on normal human fixation duration (Qvarfordt, 2017) and $\alpha=0.05$, however, these can be adjusted depending on the particular application.

\subsection{Visual Acuity}

The model from Singh et al. (2018) assumes that one fixation point represents only one target area. However, when people deliberately intend to deceive, they can use indirect vision to avoid looking at their true goal, but can also see it to claim the true goal accurately. This is because although the people do not keep their fixation points located at the true target, they can use parafoveal, perifoveal and peripheral vision to see the target outside the foveal vision (Duchowski, 2018). In this section, we propose the visual acuity function that considers whether goals are valid depending on where they are located outside of the foveal vision.

The function to calculate the visual angle of two fixation points is:

$$
\theta=2 \tanh \left(\frac{d}{2 D}\right)
$$

where $\theta$ is the angular distance between the gaze point and the target point $(\% / s), d$ is the distance in pixels between the gaze point and the target point, $D$ is the distance in pixels between the screen and the eyes. 


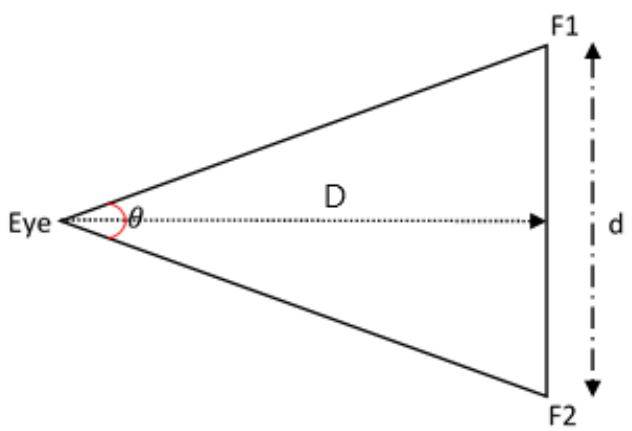

Figure 3: Illustration of the visual angle $\theta$ based on Equation 8. F1 and F2 are the two fixation points.

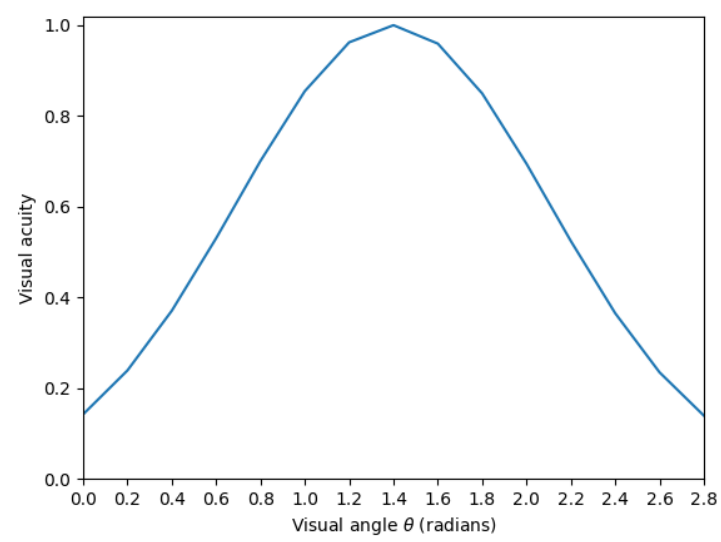

Figure 4: Illustration of the visual acuity function based on Equation 9

The visual acuity function $f v(\theta)$ illustrates the probabilities of the intentions outside the foveal vision as follows:

$$
f v(\theta)=\frac{1}{e^{(\theta-C)^{2}}}
$$

where $C$ (radians) is the visual angle threshold, which represents the maximum visual angle that our vision can see the object. In our study, since the peripheral vision's maximum visual angle is $80^{\circ}$, we choose $C=\pi \times 80 / 180 \approx 1.4$ (radians).

The visual acuity function increases the probability for intentions around the gaze point, then decreases after the visual angle threshold $C$. In this case, $f v(\theta) \rightarrow 0$ as $\theta \rightarrow \infty$ and $f v(\theta) \rightarrow 1$ as $\theta \rightarrow C$. Substitute the function of $\theta$ as above, we propose the visual acuity formula based on the Euclidean distance between two points $(d)$.

$$
f v(d)=\frac{1}{e^{\left(2 \tanh \left(\frac{d}{2 D}\right)-C\right)^{2}}}
$$

Singh et al. (2020) also defined a different version of the visual acuity function in the previous work, which they called the foveal vision function. However, their function cannot increase the fixation duration and count outside the foveal vision as they define the acuity is greatest at the location of a gaze point and then decreases the acuity as the distance $d$ increases. On the other hand, we define the maximum value of the visual acuity at the visual angle threshold $C$ (Figure 4). The reason for this is that the deceptive person would try to avoid looking at the real goal. Thus, the location of the deceptive gaze point is not the real goal in most cases. Therefore, if the visual acuity is greatest at the location of a gaze point, the fixation duration and count of the bogus goal would be maximum. So we re-define the visual acuity in this paper to show that the visual acuity of the deceptive person is minimum at the gaze point, then increases gradually up to $C$, which represents the maximum visual angle that our eyes can see the object. When the distance is greater than $C$ (i.e. $d>C$ ), we cannot see anything so the visual acuity reduces.

Using the visual acuity function $f v(d)$, we define new fixation duration and count as shown in Equation 11 and Equation 12. 


$$
\begin{gathered}
\text { deceptive_duration }_{i}=\sum_{j=1}^{\text {count }_{i}} \text { duration }_{i, j}+f v\left(d_{i, a}\right) \cdot \\
\left(1-h\left(\text { natural_duration }_{a}\right)\right) \cdot \sum_{j=1}^{\text {count }_{a}} \text { duration }_{a, j} \\
\text { deceptive_count }_{i}=\text { count }_{i}+f v\left(d_{i, a}\right) \cdot\left(1-h\left(\text { natural_duration }_{a}\right)\right) \cdot \text { count }_{a}
\end{gathered}
$$

where $i, a$ are intentions, $d_{i, a}$ is the distance between regions that infers intention $i$ and $a$, for example, the region of an intention in navigational game is the cell where the gaze point located, $j$ is the gaze point index, $1 \leq j \leq$ count $_{i}$; deceptive_duration ${ }_{i}$ and deceptive_count ${ }_{i}$ are the proposed fixation duration and fixation count of intention $i$ for deception models.

Fundamentally Equation 11 and 12 allow us to measure the strength of a person's interest in an intention by considering not just how many times they look at a target that signals it (counts), but also the times they look at the target's area. The same change is applied for how long they look at the target (duration). In short, intention $a$ is inferred from the current gaze point, while intention $i$ is not directly inferred by the current gaze point. Equation 11 increases the fixation duration of the intention $i$, which is indirectly inferred by nearby areas, by adding the fixation duration of intention $a$. The increase of the fixation duration for $i$ is based on how far it is from the intention $a\left(f_{v}\left(d_{i, a}\right)\right)$, and how much deception is happening in the game or task. If intention $a$ is likely deceptive (low $h\left(\right.$ natural_duration $\left.\left._{a}\right)\right)$, then there is a greater increase in the fixation duration of intention $i$, that we get by taking a product of $f_{v}\left(d_{i, a}\right)$ and fixation duration of $a$. Equation 12 also increases the fixation count of the intention $i$ in the same way as Equation 11.

\subsection{Models}

In this section, we present three new models of goal-recognition under deception: (1) a gazeonly model that uses the visual acuity measure; (2) a modulated gaze-only model that uses the honesty degree to moderate confidence in predictions; and (3) a model that combines the gaze-only models with a model-based intention recognition algorithm that uses ontic actions.

Deception Gaze Model (DG) Using Equation 11 and Equation 12, we define our first gaze model, Deception Gaze, as follows:

$$
s d_{i}=\lambda \cdot \text { deceptive_duration }_{i}+(1-\lambda) \cdot \text { deceptive_count }_{i}
$$

where $\lambda$ is the relative weight as defined in Equation 4. We do not use the log function as in Equation 4 because Singh et al. (2018, 2020) applied the log function to limit the importance of old intentions. They argued that the order of intentions are less relevant since people change their intentions over time. However, in the deceptive scenarios, people would have to find the real goal at the beginning of the task and deliberately hide their real intentions since then. Therefore, the early intention is the most reliable one so the log function should not be applied in Equation 13. 
We then normalise the fixation score function in Equation 13 to get the posterior probability of an intention $i$ :

$$
P_{D}\left(i \mid O_{g}\right)=\frac{s d_{i}}{\sum_{j \in I} s d_{j}}
$$

Modulated Deception Gaze Model (MDG) We can modulate the posterior probability of the Deception Gaze model by multiplying the probabilities with the honesty degree resulting in our second gaze model, the Modulated Deception Gaze. The modulation can be seen as the ability of the model to moderate its confidence in the predictions; that is, the posterior probabilities, similar to the strategy proposed by Masters and Sardina (2019). Intuitively, the more the deceptive a person's gaze, the more uniform the posterior probabilities. Formally:

$$
P_{M D G}\left(i \mid O_{g}\right)=\kappa \cdot h\left(\text { natural_duration }_{i}\right) \cdot P_{D}\left(i \mid O_{g}\right)
$$

where $P_{D}\left(i \mid O_{g}\right)$ is the posterior of deception gaze-only, calculated in Equation 14, $\kappa$ is the normalising constant to ensure that all probabilities add up to $1, h$ (natural_duration $_{i}$ ) is the honesty degree of intention $i$ based on the fixation duration in Equation 7, and $P_{M D G}\left(i \mid O_{g}\right)$ is the modulated posterior probability of intention $i$. Note that, h(natural_duration $i$ ) in Equation 15 is responsible to modulate the confidence. That means when the goal seems to be bogus, its confidence will be lower. Whereas $\left(1-h\left(\right.\right.$ natural_duration $\left.\left._{a}\right)\right)$ (Equation 11 , 12) in the deceptive count and duration formulas is for increasing the fixation count and duration within the peripheral vision in case the gaze is deceptive (i.e. low honesty degree).

Deception Gaze+Ontic Model (DGO) Based on Natural Gaze+Ontic model defined in Equation 6, the posterior probability of Deception Gaze+Ontic is calculated as follows:

$$
P_{D G O}\left(i \mid O_{a}, O_{g}\right)=\kappa \cdot P\left(O_{a} \mid i\right) \cdot P_{D}\left(i \mid O_{g}\right)
$$

in which $P_{D}\left(i \mid O_{g}\right)$ is Equation 14, $P\left(O_{a} \mid i\right)$ is the likelihood of an intention $i$, defined in Equation 2 where $\beta=1$ (Masters \& Sardina, 2017a), $\kappa$ is the normalising constant. $P\left(O_{a} \mid i\right)$ is the probability of observing actions $O_{a}$ if $i$ was the intention of the agent, calculated using any method for model-based goal recognition (Vered et al., 2016).

Modulated Deceptive Gaze+Ontic (MDGO) We can also modulate the Deception Gaze+Ontic probability by substituting $P_{M D G}$ for $P_{D}$. We then have Modulated Deceptive Gaze+Ontic model as follows:

$$
P_{M D G O}\left(i \mid O_{a}, O_{g}\right)=\kappa \cdot P\left(O_{a} \mid i\right) \cdot P_{M D G}\left(i \mid O_{g}\right)
$$

Modulated Deception Gaze+Modulated Ontic (MDGMO) We can further modulate the ontic-action by assigning $\gamma=1$ in Equation 1. We call this Modulated Deception Gaze+Modulated Ontic. The MDGO model that involves the honesty degree is more robust under deception. However, when we include the rationality measure $\beta$ (Masters \& Sardina, 2019), the MDGMO model does not perform better than the MDGO model (Section 5). Masters and Sardina (2019)'s model is good for irrationality, but not necessarily under deception, which is intentional. The reason for including $\beta$ does not improve the performance 
of MDGMO is that $\beta$ is a measure to flatten out the probability distribution in the case of irrationality. But $\beta$ cannot increase the probability of the real goal.

\subsection{Quantifying Deception}

Besides the main purpose of modulating the confidence of candidate intentions in Section 3.2, the honesty degree of all intentions at a single time step can also be used to: 1) distinguish between different deceptive gaze strategies; and 2) quantify gaze deception without knowing the goals. Besides the fixation duration, we use the Euclidean distance between the ontic-action and the gaze point as another useful feature to formulate honesty degree. Note that we use the fixation duration as a parameter to measure the honesty degree of a single intention. On the other hand, we use both the fixation duration and the distance between the fixation point and the location of ontic-action to calculate the honesty degree of all intentions at a time step.

We expect a person's gaze to follow or be close to their source of physical actions, particularly when people are deliberately performing actions. For example, consider someone playing a racing car game. They have to concentrate and keep their eyes focus on their moving car. Based on this, we define the honesty degree based on the distance between the fixation point and location of the ontic-action:

$$
h\left(\text { distance }_{t}\right)=\frac{1}{1+e^{\alpha\left(\text { distance }_{t}-\text { DistanceThreshold }\right)}}
$$

where distance $_{t}$ (pixels) is the average distance between the gaze point and the location of current action at time step t; Distance Threshold is the distance threshold to ensure that at the threshold point, the honesty degree is 0.5 ; and $\alpha$ is also a rate parameter. In our studies, we chose DistanceThreshold $=150$ (pixels) $(39.69 \mathrm{~mm})$, and $\alpha=0.05$.

The average honesty degree of all intentions based on fixation duration at time step $t$ is:

$$
\bar{h}\left(\text { step_duration }_{t}\right)=\frac{1}{\left|I_{t}\right|} \sum_{i \in I_{t}} h\left(\text { step_duration }_{i}\right)
$$

where $I_{t}$ is a set of all intentions at time step $t$; $h\left(\right.$ step_duration $\left._{t}\right)$ is the honesty degree in Equation 7 where step_duration $_{i}$ represents the accumulated fixation duration of intention $i$ at time step $t$, defined as follows:

$$
\text { step_duration }_{i}=\sum_{j \in G_{t}} \text { duration }_{i, j}
$$

where $G_{t}$ is a set of gaze points up to time step $t$.

Based on these two expectations around natural gaze behaviours, that is, using the notions of fixation duration and distance between gaze-point and ontic-action, we define $H_{t}$ below that we use to quantify deception.

$$
H_{t}= \begin{cases}\bar{h}\left(\text { step_duration }_{t}\right) & \left(t-t_{0}\right)<X \\ \min \left(\bar{h}\left(\text { step_duration }_{t}\right), h\left(\text { distance }_{t}\right)\right) & \text { Otherwise }\end{cases}
$$

where $\left(t-t_{0}\right)$ is the time difference between the beginning of the task (e.g. game) $\left(t_{0}\right)$ and the current time step $(t)$, therefore the distance feature may not be as important to 


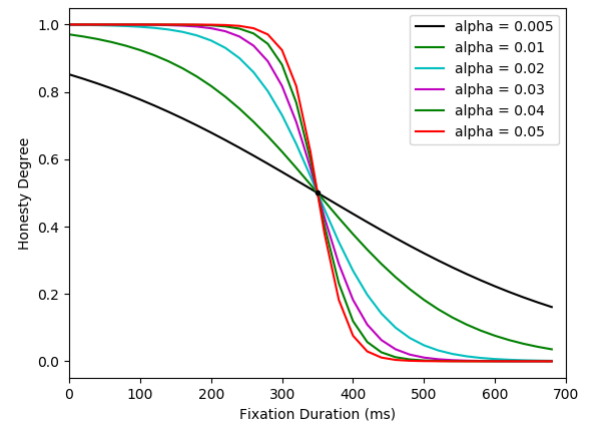

(a) The threshold point is $(350,0.5)$

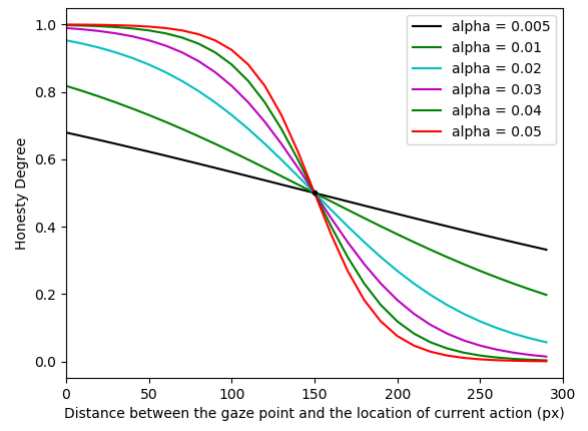

(b) The threshold point is $(150,0.5)$

Figure 5: The rate parameter changes the shape of the sigmoid function

determine the deceptive behaviour during this period. The time of planning is dependant on the domain and the experience of the agent, that is, $X$. In our study, we choose $X=2(s)$.

Note that while Equation 7 represents the honesty degree of one intention at each time step, Equation 20 defines the honesty degree of all intentions at each time step. We use Equation 20 to detect and quantify deception by measuring the honesty degree at each time step. We use Equation 7 for our models defined in Section 3.3 and 3.4.

To quantify deception, we define the deceptive magnitude, which is calculated using the average degree of honesty of all time steps, defined as follows:

$$
\text { deceptive_magnitude }=1-\frac{1}{n} \sum_{t=1}^{n} H_{t}
$$

where $n$ is the total number of time steps and $H_{t}$ is Equation 20.

\subsection{The Effect of Rate Parameter}

Figure 5 shows how changing the rate parameter $\alpha$ affects the shape of the honesty degree. There is no "optimal" rate parameter $\alpha$. Based on the purposes of the honesty degree, changing the rate parameter will not change the results when using the honesty degree as a measure to detect deception because the threshold of the sigmoid function is always 0.5 regardless the value of the rate parameter. Also, the expected accuracy of $D G$ model and $D G O$ model will not change. However, it affects the honesty degree to modulate probability distribution, that is, the measures of $M D G$ model and $M D G O$ model.

\section{Experiment Design}

In this section, we present the design of our two experiments, which includes data from the games of two human-subject experiments. 


\subsection{Case Study 1: Single-Player Navigational Game}

\subsubsection{Conditions And Game Design}

In our first case study, we created a straightforward navigational game that required minimal training of participants, and is based on the VIP problem discussed in Masters and Sardina (2017b). In this game, players attempt to go to a specific goal from a starting point. They can move either horizontally or vertically in a grid-based map. All moves cost 1 . The game is designed in JavaScript language and can be played in a web browser ${ }^{1}$. Each player plays three rounds with 10 maps per round in the order below. Each round has a specific instruction that will impact the player's gaze and ontic strategies. Before the experiment, the players' gaze was calibrated using the default calibration of the eye tracker. Therefore, the players knew that their gaze was being tracked. But they did not know it was being used to infer their intentions so they had no incentive to deliberately change their gaze actions. Three study conditions are illustrated as follows:

1. Condition 1 (Natural Gaze + Ontic Action): In the first round, the participants were told to go to the goal. The condition served as a baseline to record the natural actions of the player.

2. Condition 2 (Natural Gaze + Deceptive Ontic Action): In the second round, the participants were told to find the path to the real goal as if they were trying to deceive an observer who did not know the real goal. At this stage, they were not aware that their gaze was being tracked and used to infer intentions. Therefore, the objective of this condition is to record the deceptive path-planning, but with natural gaze.

3. Condition 3 (Deceptive Gaze + Deceptive Ontic Action): In the third round, the participants were again told to deceive the path, but also that their gaze was being tracked and would be used to infer their intentions. Therefore, they would know that they can manipulate their gaze and intentionally mislead through both ontic moves and gaze.

\subsubsection{MAP DESIGN}

There are 10 maps per round. The first 5 maps are the same in all three conditions, while the last 5 maps are different between conditions. In total, we designed 20 different maps. We varied some map parameters to give non-uniform maps, including:

- number of goals ranges from three to six;

- size of the map is either small $(10 x 10)$ or big $(13 \times 13)$;

- distance between different goals - the goals are designed to be either close or far from each other;

- number of obstacles is either none or many; and

1. https://github.com/thaole25/vip-routing 
- number of different ways to reach the goal from the starting point - some maps are designed to have only one way to achieve the goal.

Each tile is a square of size 58 pixels. The border between two adjacent tiles has a size of 12 pixels. This gives enough space between tiles to take accurate readings of which tile people's gaze was focused on. Figure 1 in the introduction shows one of the simplest maps used in our experiment.

\subsubsection{Case Study 1 Data COllection}

The study was conducted in a university lab, with one player in each session. The resolution of the screen is $(1920 \times 1080)$ and the diagonal is approximately $58 \mathrm{~cm}$. Thus, the PPI is about 96 pixels per inch. The computer is fitted with a Tobii Pro X3-120 eye tracker $(120 \mathrm{~Hz})^{2}$. We also used Tobii Studio software to record the entire study session and generate the gaze data for later analysis. The log file of gaze contains (x, y) coordinate of the fixation, the fixation duration and the timestamp of the fixations.

At the beginning of the study, the participant was allocated to the university lab with one researcher. They were also given a written document of the study and consent form. Following this, the participants' eyes were calibrated using default calibration of the eye tracker and Tobii Studio software. After this, the researcher would give a brief introduction about the game. The game was started by accessing to an URL in the web browser. There are three rounds for the game. For each round, the players were requested to play a tutorial until they understood the game. In this tutorial, before each round (condition), the players were asked if they understood the requirements of this round (condition) and then a map was shown (different to other maps in the game) and they were told if they can press the key (up, down, left, right) to reach the goal. Participants then play a game of three rounds with 10 different maps for each round. During the game session, the moves of the players were also recorded and stored for later analysis. The movement $\log$ file contains $(\mathrm{x}, \mathrm{y})$ coordinate and timestamp of each move. In total, we recruited 30 players $(8 \mathrm{~F} / 22 \mathrm{M})$ from the same university, aged between 22 and 35, resulting in 30 (participants) $\times 10$ (games) $=300$ (games) in each condition, which makes a total of 900 (data points) for all three conditions.

\subsection{Case Study 2: Ticket To Ride Multi-Player Game}

In this section, we describe our second study on a multi-player game called Ticket to Ride (TTR) - a competitive multi-player game in which deception can be used by players to gain advantages against their opponent.

\subsubsection{Ticket TO Ride}

We employed the use of a multi-player game called Ticket to Ride $^{3}$ (TTR) to elicit human intention in a controlled environment, as used in previous works (Newn et al., 2017, 2018; Singh et al., 2018). A screenshot of this game is shown in Figure 2.

The goal of this game is to build routes between cities across the United States and southern Canada. At the beginning of the game, the player picks either two or three

2. https://www.tobiipro.com/product-listing/tobii-pro-x3-120/

3. http://www . daysof wonder. com/tickettoride/en/ 
Destination Ticket Cards, each of which has a pair of cities that they need to build a route to connect. In each turn, the player has an option of either draw a new card or place a train card on the map. When the player finishes a route, he receives points assigned in the ticket card. Otherwise, he loses the same number of points if he cannot connect the destinations before the game ends. A player can block their opponent's route as each route can only be claimed by one player. Therefore, a player can gain significant advantage by inferring his opponent's path. We chose this game as one of our studies because a player can infer his opponent's objectives by looking at their gaze.

\subsubsection{Case Study 2 Data}

The data was collected and used by Newn et al. (2018) and Singh et al. (2020). The study was conducted in a university lab, with two players in each session. A total of 40 participants were recruited and randomly assigned into 20 pairs of players. At the beginning of the study, the players were given a written overview of the study and consent form. After this, participants played the tutorial that comes with the Ticket To Ride game to ensure they understood the rules. This shows the players where to choose cards, how to place the card on the route, etc. The map in this tutorial is different from the map on which they play the real game. Participants then played a short practice round to guarantee that they are comfortable with the game. Each pair was required to play two rounds of the game against each other. Each round corresponds to a condition that we want to test. The two conditions are explained as follows.

1. Condition 1: Natural Gaze: The gaze of the unaware player was tracked. They were unaware that the gaze was being displayed to their opponent. This condition was designed to record the natural gaze of the players.

2. Condition 2: Deceptive Gaze: The gaze of the aware player was tracked. Aware players deliberately altered their gaze and they were aware that their opponent (the unaware player) could see their gaze. The unaware player was not told that their gaze was being displayed to the aware player. The objective of this condition was to record the deceptive gaze when the aware players attempted to manipulate their gaze behaviour.

In total, we had 40 game data corresponds to 40 participants with an average of 21 minutes per game.

\subsection{Independent Variables}

We evaluated five models, which are:

1. Deception Gaze $(D G)$ : Equation $14, P_{D}\left(i \mid O_{g}\right)$

2. Modulated Deception Gaze (MDG): Equation 15, $P_{M D G}\left(i \mid O_{g}\right)$

3. Deception Gaze+Ontic (DGO): Equation 16, $P_{D G O}\left(i \mid O_{a}, O_{g}\right)$

4. Modulated Deception Gaze+Ontic $(\beta=1)(M D G O)$ : Equation $17, P_{M D G O}\left(i \mid O_{a}, O_{g}\right)$ 
5. Modulated Deception Gaze+Modulated Ontic (MDGMO) (with $\gamma=1$ in Equation 1)

We compare the five models with with the three baseline models from Singh et al. (2018): Ontic (O), Natural Gaze (G) and Natural Gaze+Ontic (GO).

\subsection{Measures}

To evaluate the results and compare the different models as proposed, we used the following measures:

1. Accuracy: This evaluates how successful our model is when predicting the real goal of the player. Accuracy is measured based on two metrics:

- Precision/Recall Curve is the precision against recall plot. We choose this measure because (1) the output is a probabilistic value, therefore we can measure the performance of models without a classification threshold and (2) the data are highly imbalanced between the real and the bogus goals as there is only one real goal and multiple bogus goals in one map in the first study. Thus, using Precision/Recall Curve is suitable when we focus on the performance of predicting the real goal.

- Average ground truth probability is calculated based on the probability of the real goal at every time step. The formula to calculate this measure is defined as follows:

$$
a_{c}=\frac{1}{\left|Z_{c}\right|} \sum_{z \in Z_{c}}\left(\frac{1}{\left|T_{z}\right|} \sum_{t \in T_{z}} P\left(g_{r}\right)_{z, t}\right)
$$

where $a_{c}$ is the accuracy of condition $c, Z_{c}$ is a set of games in condition $c, T_{z}$ is the set of time steps in game $z, P\left(g_{r}\right)_{z, t}$ is the probability that the model assigned to the real goal at time step $t$ in game $z$.

Note that in the first study, ground truths are the real goals and non ground truths are the bogus goals. In the second study, ground truths are the routes of the tracked player that they claim. Non ground truths are all other routes and other targets, such as opponent's profile, drawn tickets, drawn cards, own profile, tickets and possible cards to select from.

2. Inference horizon (Singh et al., 2018): This measures the time in seconds between when the game ends and when a model begins to return the correct prediction for the remainder of the game. Higher inference horizon implies earlier prediction.

\section{Results}

In this section, we present the results from our experiments.

\subsection{Single-Player Navigational Game}

\subsubsection{ACCURACy AND InfEREnCE Horizon}

We describe the accuracy summary for all models as shown in Figure 6, 7 and 8 . The results are summarised in Table 1 and the Wilcoxon signed-rank test is shown in Table 3 


\begin{tabular}{lrrrrrrrr}
\hline & \multicolumn{2}{c}{ Condition 1 } & & \multicolumn{2}{c}{ Condition 2 } & & \multicolumn{2}{c}{ Condition 3 } \\
\cline { 2 - 3 } & PRC & Prob & & PRC & Prob & & PRC & Prob \\
\hline O & $\mathbf{0 . 8 2 9}$ & 0.62 & & 0.319 & $\mathbf{0 . 3 5}$ & & $\mathbf{0 . 4 2 7}$ & $\mathbf{0 . 4 0}$ \\
MO & $\mathbf{0 . 8 3 1}$ & 0.57 & & 0.292 & 0.34 & & 0.398 & 0.37 \\
\hline G & 0.758 & 0.62 & & $\mathbf{0 . 4 2 3}$ & $\mathbf{0 . 3 8}$ & & 0.232 & 0.24 \\
DG & 0.498 & 0.42 & & 0.350 & $\mathbf{0 . 3 5}$ & & 0.268 & 0.28 \\
MDG & 0.497 & 0.43 & & 0.385 & $\mathbf{0 . 3 7}$ & & 0.326 & 0.33 \\
\hline GO & 0.697 & $\mathbf{0 . 6 4}$ & & 0.377 & $\mathbf{0 . 3 8}$ & & 0.284 & 0.30 \\
DGO & 0.775 & $\mathbf{0 . 6 5}$ & & 0.325 & $\mathbf{0 . 3 8}$ & & 0.409 & 0.40 \\
MDGO & 0.753 & $\mathbf{0 . 6 4}$ & & 0.336 & $\mathbf{0 . 3 9}$ & & $\mathbf{0 . 4 2 5}$ & $\mathbf{0 . 4 1}$ \\
MDGMO & 0.723 & 0.60 & & 0.328 & $\mathbf{0 . 3 7}$ & & 0.388 & 0.37 \\
\hline
\end{tabular}

Table 1: Accuracy of the navigational game: $P R C$ is the area under the curve of the precision-recall curve, Prob is the average ground truth probability. Condition $1=$ Natural Gaze, Natural Ontic; Condition 2 = Natural Gaze, Deceptive Ontic; Condition $3=$ Deceptive Gaze, Deceptive Ontic. Winners are highlighted (including the results that are insignificantly different).

and Table 4. We applied the Wilcoxon signed-rank test for the statistical hypothesis test because this is a non-parametric test used when the data is not normally distributed. We tested the data normality using the Shapiro-Wilk test and found that our data was not normally distributed. More details for the Wilcoxon signed-rank test can be found in Appendix A.

Table 1 shows the results of all models in the three conditions. Overall, using two metrics (PRC and the average ground truth probability), we can see that $O$ and $M D G O$ have the best performance among other models. However, in condition 1 (natural condition), $O$ model still offers higher accuracy than other models. Moreover, $M O$ model is slightly worse than $O$ model. Also, $M D G M O$ model does not offer higher accuracy than the $M D G O$ model.

For more details, in Figure 6, the previous model $G$ performs better in condition 1 and 2 (natural gaze data) than our models. However, our model $M D G$ offers slightly higher area under the curve (0.326) than $G$ model (0.232) in condition 3. To compare between different Gaze+Ontic models, as shown in Figure 7, in condition 1 and 2 (natural gaze data), MDGO performs similarly to $G O$ model. However, in condition 3 (deceptive gaze data), MDGO has significantly higher area under the curve (auc) value (0.425) compared to $G O$ model (0.284). When comparing to $O$ model, $M D G O$ model performs similarly in condition 2 and 3 (deceptive ontic data). But $M D G O$ is insignificantly worse than $O$ model when running on condition 1 with natural ontic data.

Figure 8 shows the average ground truth probability for eight models to evaluate how different models change the absolute probabilistic value of the real goal. First, we consider three models for gaze: Natural Gaze $(G)$, Deception Gaze $(D G)$ and Modulated Deception Gaze (MDG). Our $D G$ offered significantly higher probability (28\%) than $G(24 \%)$ $(p<0.05, d=0.25)$ in predicting the real goal when the gaze behaviour is dishonest in con- 

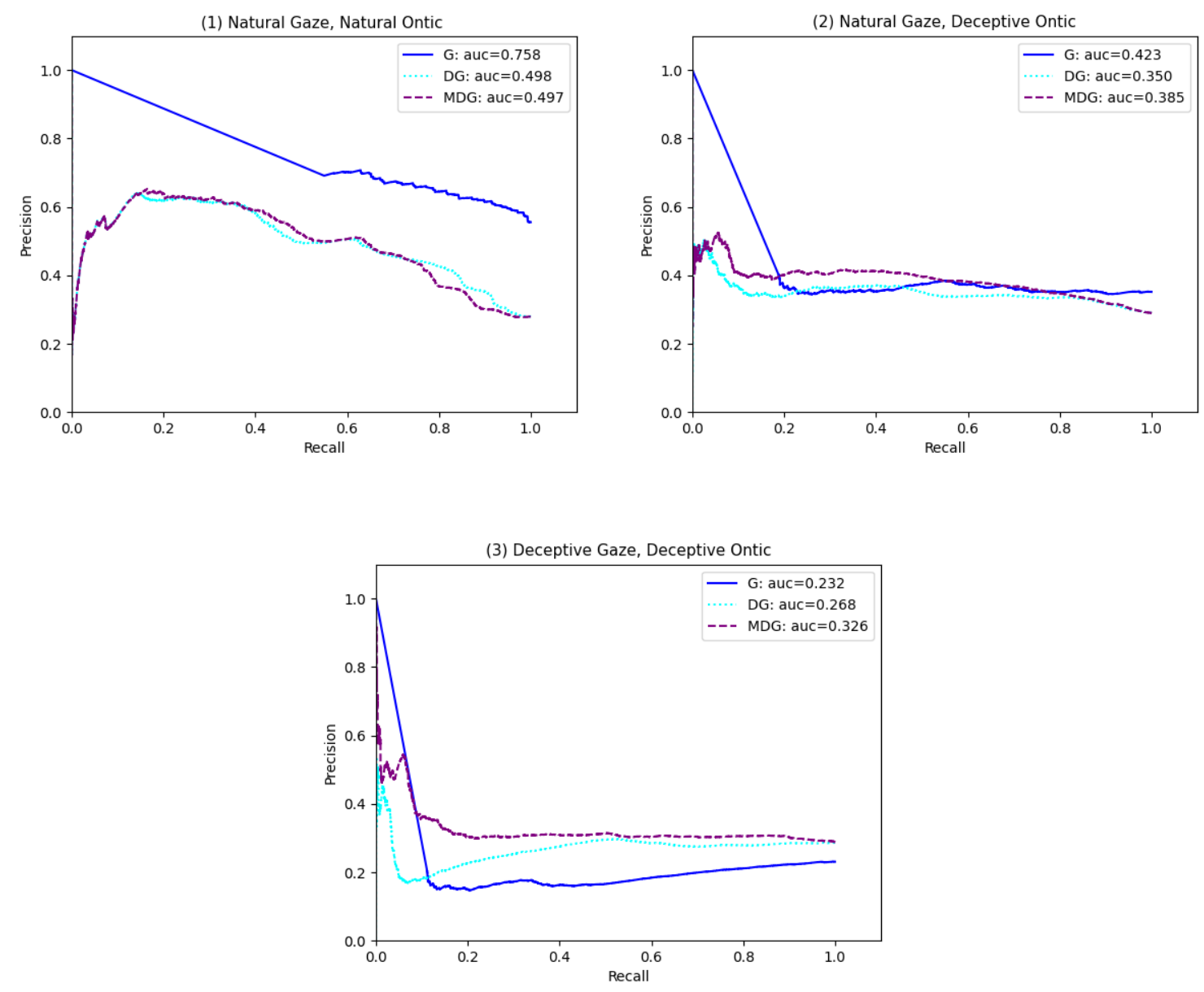

Figure 6: Case study 1: Precision/Recall Curves in three condition of three models: $G=$ Natural Gaze, DG = Deception Gaze, $M D G=$ Modulated Deception Gaze. The labels are the area under the curves (AUCs). 

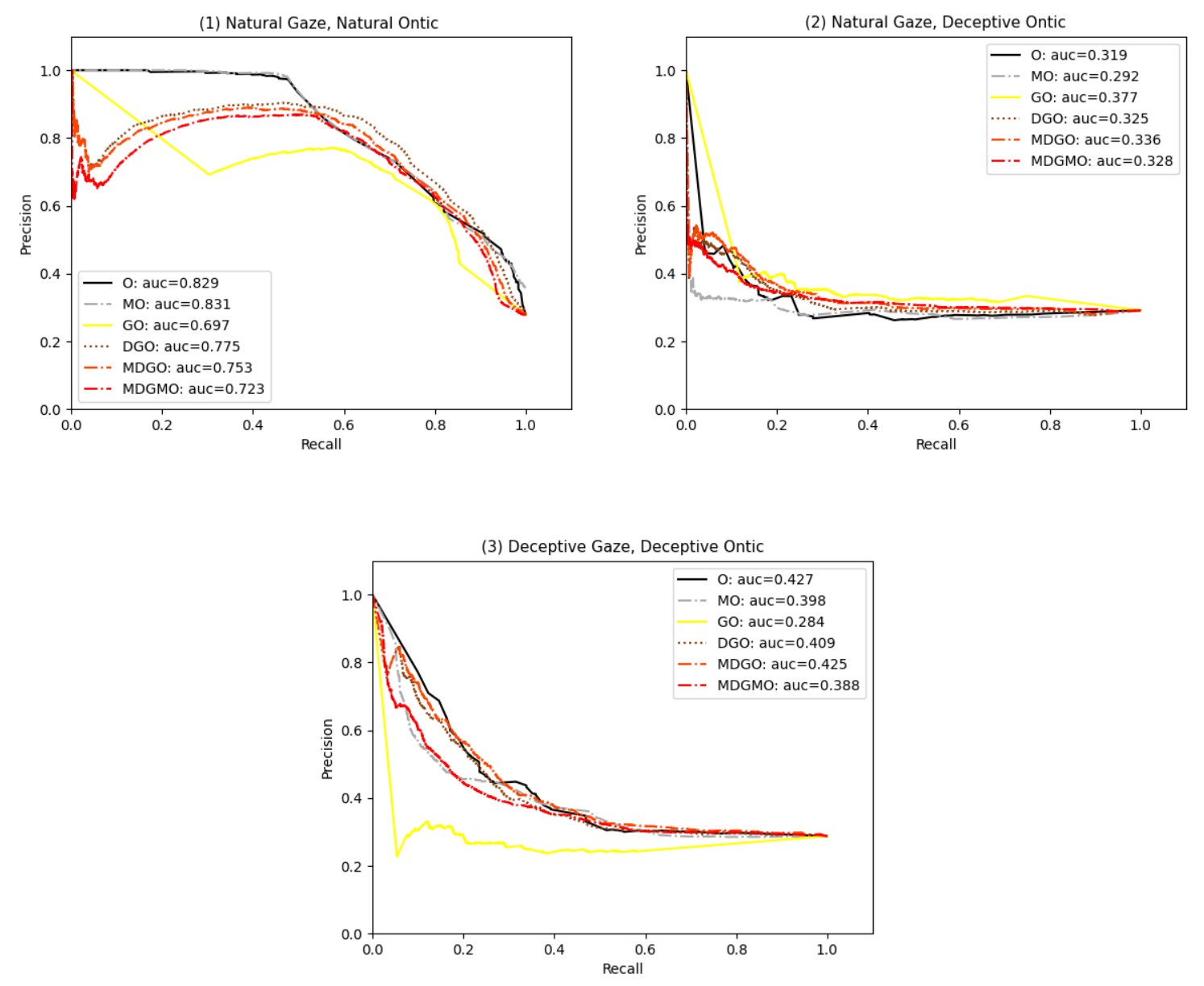

Figure 7: Case study 1: Precision/Recall Curves in three condition of 6 models: $O=O n$ tic, $M O=$ Modulated Ontic, GO = Natural Gaze+Ontic, DGO = Deception Gaze+Ontic, $M D G O=$ Modulated Deception Gaze+Ontic, MDGMO = Modulated Deception Gaze + Modulated Ontic. The labels are the area under the curves (AUCs). 


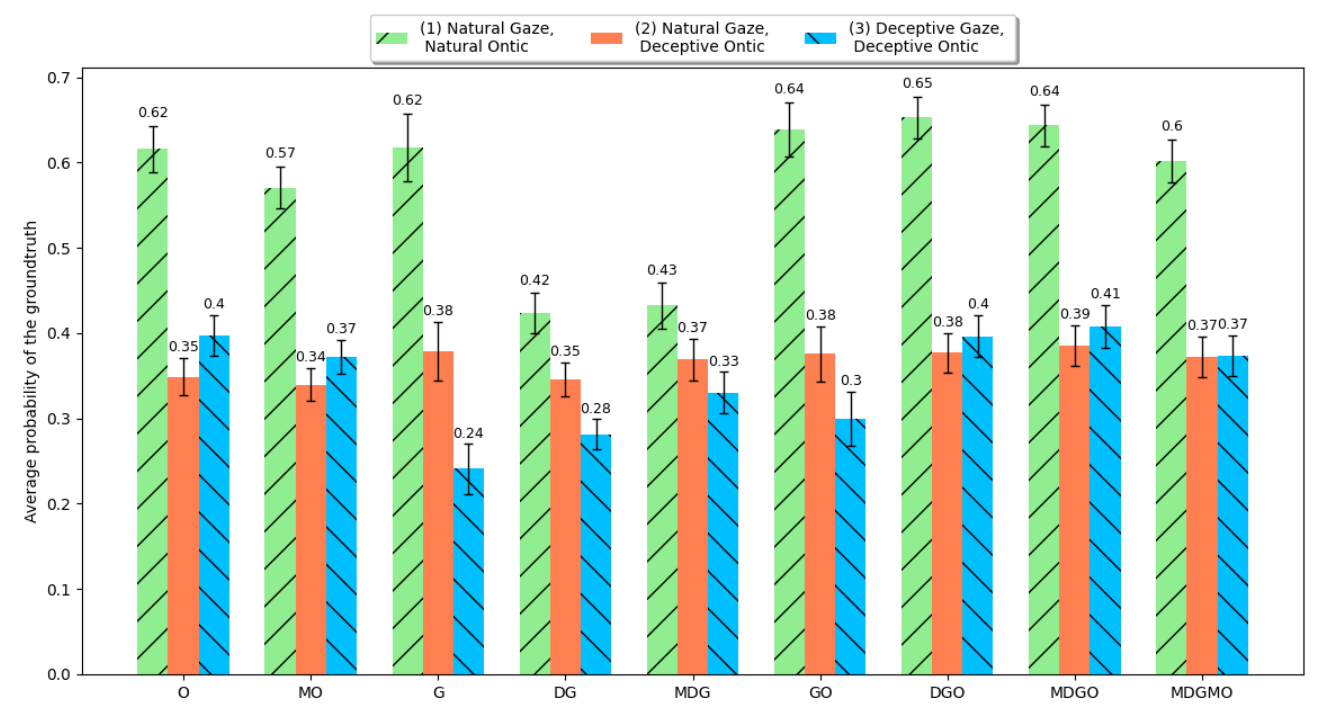

Figure 8: Case study 1: Average probability of the ground truth for 9 models in 3 conditions: $O=$ Ontic, $M O=$ Modulated Ontic, $G=$ Natural Gaze, DG $=$ Deception Gaze, MDG $=$ Modulated Deception Gaze, GO = Natural Gaze+Ontic, DGO = Deception Gaze+Ontic, $M D G O=$ Modulated Deception Gaze+Ontic, MDGMO = Modulated Deception Gaze + Modulated Ontic. The error bars show 95\% confidence interval.

dition 3. Moreover, in this condition, our $M D G$ model offered the best probability (33\%). In addition, Wilcoxon signed-rank test shows that this model has significantly better performance than $D G$ model $(p<0.05, d=0.35)$ and $G(p<0.05, d=0.39)$ in condition 3. However, $M D G$ performed significantly worse in condition 1 than $G$ and $D G$. In condition 2 , there is insignificant difference in the ground truth probabilistic value between $M D G$ and $G(p=0.6, d=0.03)$. There is also minimal difference between $D G$ and $G$ in condition 2 $(p=0.05, d=0.12)$.

Considering four Gaze+Ontic models: Natural Gaze+Ontic (GO), Deception Gaze+Ontic (DGO), Modulated Deception Gaze+Ontic (MDGO) and Modulated Deception Gaze+Modulated Ontic (MDGMO). Our enhanced models DGO and MDGO did not improve the ground truth probability in condition 1 and 2, in which we have natural gaze behaviours; but the differences of these two models with $G O$ in both natural conditions is insignificant $(p>0.05, d \leq 0.14)$. Moreover, $M D G O$ offered significantly better probability $(41 \%)$ of the real goal when agents intentionally altered their gaze actions in condition 3 than $G O$ model $(30 \%)$. In condition 3, Wilcoxon signed-rank test shows that there is minimal difference between MDGO (41\%) and DGO (40\%) $(p=0.01, d=0.15)$, also between $M D G O$ and $O$ $(p>0.05, d=0.0)$. Regarding the $M D G M O, M D G O$ offers significantly higher probability in all conditions $(p<0.05, d \geq 0.2)$. Therefore, modulating ontic action by setting $\gamma=1$ in Equation 1 did not improve the results. Overall, Gaze+Ontic models offered a higher probability than Gaze models in all three conditions, while the Ontic models only offered higher accuracy than Gaze models in conditions 1 and 3. MO model performs slightly 


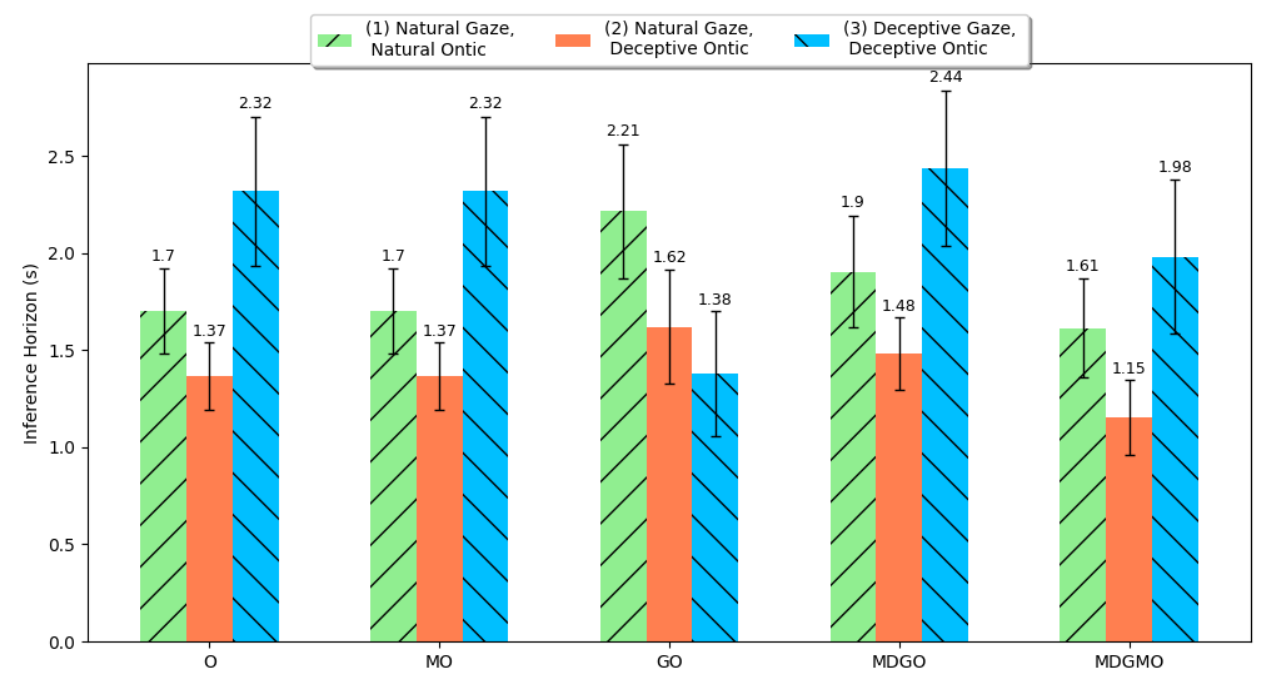

Figure 9: Case study 1: Inference horizon of 5 models Ontic (O), Modulated Ontic (MO), Natural Gaze+Ontic (GO), Modulated Deception Gaze+Ontic (MDGO) and Modulated Deception Gaze + Modulated Ontic (MDGMO) in three conditions.

worse than $O$ model and $M D G M O$ also offers lower probability than $M D G O$ in all three conditions.

Regarding the inference horizon measures, as seen in Figure 9, $M D G O$ returned the correct prediction significantly earlier than $G O$ in condition $3(p<0.05, d=0.42)$. In the second condition, there is no significant difference between these two models $(p>$ $0.05, d=0.1)$ because the effect size $(d=0.06)$ is trivial. Also, when the actions are honest in condition $1, M D G O$ is slightly worse than $G O(d=0.1)$. Comparing with $O$ model, $M D G O$ predicts the real goal significantly earlier than $O$ model in all conditions $(p<0.05, d>0.11)$. There is no difference between $O$ and $M O$. Also, MDGMO performs significantly worse than $M D G O$ model.

In summary, $M D G O$ model improves the accuracy significantly compared to $G O$ model, which is the previous state-of-the-art gaze-based and ontic-based model. However, MDGO offers similar accuracy to Ontic model in this case. Regarding the $M O$ and $M D G M O$ model, they do not perform better than the $O$ and $M D G O$ model. Also, MDGO returns the correct prediction significantly earlier than all other models. Thus, in this study, gaze is useful to return the prediction earlier, but not improve the accuracy overall. We will discuss later in the discussion section on how gaze is useful in different cases.

\subsubsection{Gaze Feature Analysis}

In this section, we analyse two gaze features empirically: the fixation and the distance between the gaze point and the ontic action. This analysis aims to back up our claim that these two features are important to distinguish between natural and deceptive gaze. 


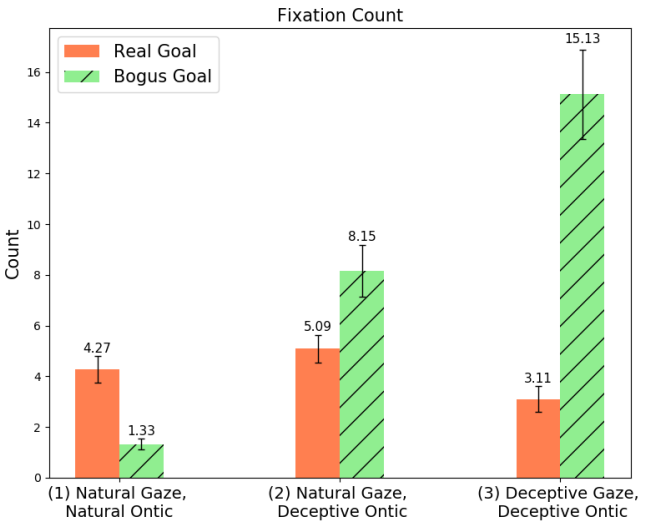

(a) The average fixation count

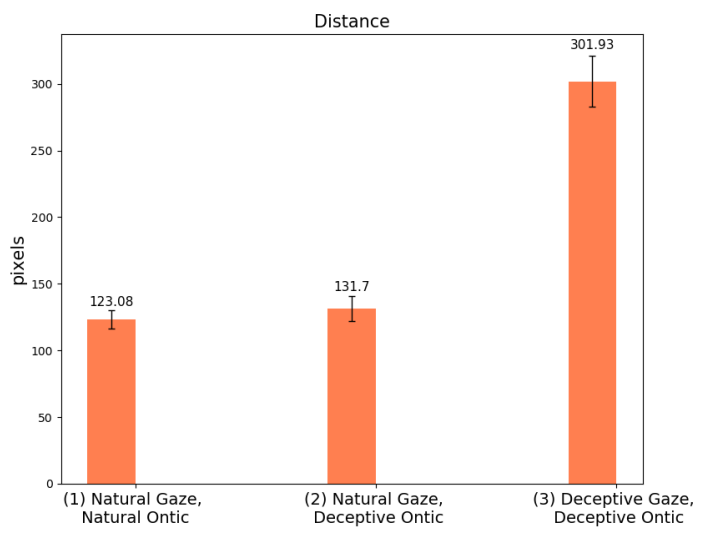

(c) The average distance between the gaze point and the moving object. (pixels)

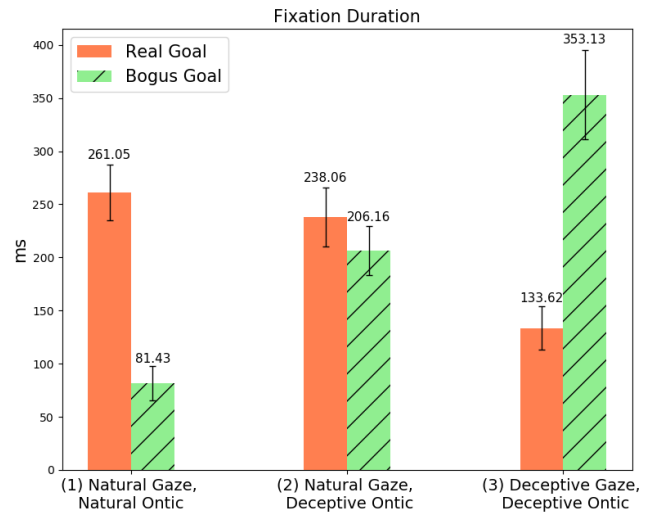

(b) The average fixation duration

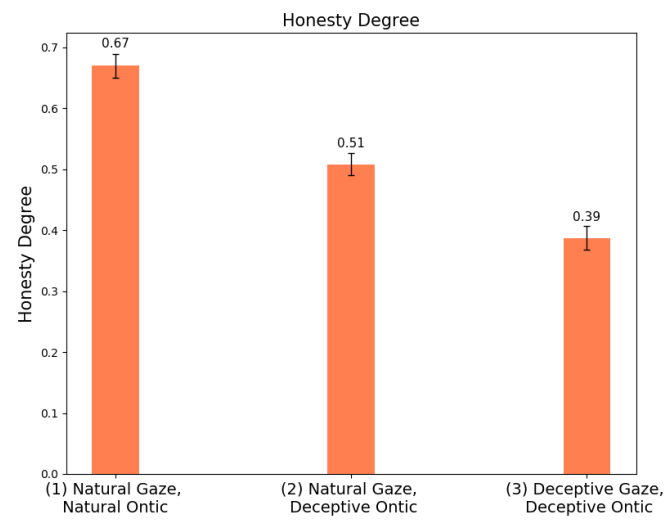

(d) The average honesty degree

Figure 10: Gaze Features in three conditions 
Figure 10a and 10b show the fixation count and fixation duration statistics at the real goal and the bogus goal. Wilcoxon signed-rank test shows that there is no significant difference of the fixation count and duration at the real goal between condition 1 and 2 $(p>0.05, d<0.1)$. This is because the gaze behaviour in these two conditions is natural. But we can see the significant difference at the bogus goal due to the player must attempt to plan a deceptive path in condition 2, which influenced the gaze to look at the bogus goal more often. To compare the natural gaze and deceptive gaze behaviours, we look for the difference between condition 2 and 3, in which we have the same ontic action. The fixation count and duration at the real goal is significantly lower for the deceptive gaze $(p<0.05, d>0.35)$; meanwhile, at the bogus goal, these two fixation features are significantly higher $(p<0.05, d>0.38)$. This result shows that the deceptive gaze is using both simulation (focusing attention on the bogus goal) and dissimulation (avoiding the real goal) strategy. Masters and Sardina (2017b) and Bell (2003) indicated that simulation involves dissimulation because focusing on the bogus goal also means avoiding looking at the real goal.

Regarding the distance between the gaze point and the ontic action in Figure 10c, the distance of natural gaze in condition 1 and 2 are almost equal ( $p>0.05, d=0.07)$. However, the distance of deceptive gaze is significantly greater than the distance of the natural gaze $(p<0.05, d=1.19)$. Thus, distance from gaze point to action is a useful feature to distinguish between natural and deceptive gaze behaviours.

Figure 10d shows the average honesty degree in three conditions based on Equation 20. The lower honesty degree implies stronger deceptive strategy. As shown in this figure, the honesty degree is significantly lower in condition 3 (deceptive gaze) than in condition 1 and 2 (natural gaze). Also, the honesty degree in the deceptive gaze condition is less than 0.5, which is the threshold of the sigmoid function that helps identify the deception. For further discussion of how the rate parameter $\alpha$ changes the shape of the honesty degree, see Section 3.6.

\subsubsection{Deceptive Gaze Strategies}

In our game, we observed three fundamental gaze deception strategies:

- Gaze Scattering: This involves players either scattering the gaze across the entire screen or all of the goals (destinations).

- Gaze Averting: This strategy is similar to both Gaze Averting and Gaze Camouflage described by Newn et al. (2018). Players try to avoid looking at the true goal by looking at the nearby locations, but not at the bogus goal. They used the peripheral vision or brief glances the true goal to achieve the goal.

- Gaze Misdirection: Here the players fixated on one of the bogus goals, increasing the probability of the bogus goal. 

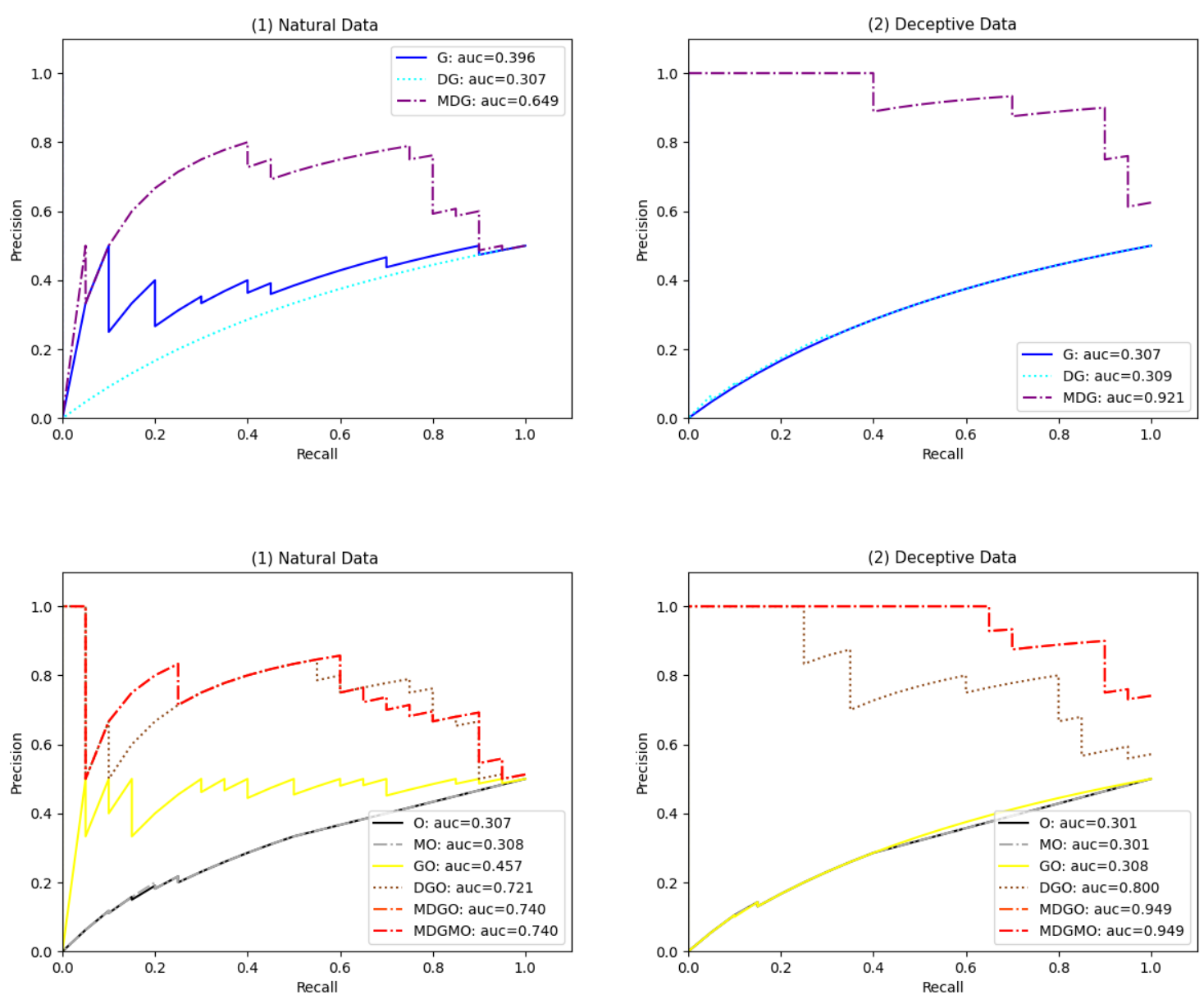

Figure 11: Case study 2: Precision/Recall Curves in two condition. 9 models: $O=$ Ontic, $M O=$ Modulated Ontic, $G=$ Natural Gaze, DG $=$ Deception Gaze, MDG = Modulated Deception Gaze, GO = Natural Gaze+Ontic, DGO = Deception Gaze+Ontic, MDGO = Modulated Deception Gaze+Ontic, MDGMO = Modulated Deception Gaze + Modulated Ontic. The labels are the area under the curves (AUCs).

\subsection{Case Study 2: Multi-Player Game}

\subsubsection{ACCuracy And Inference Horizon}

In this section, the results are summarised in Table 2. The Wilcoxon signed-rank test is shown in Table 5 and Table 6 . As we can see in this table, MDGO performs significantly better than other models in both conditions. Also, there is no difference between $M D G O$ and $M D G M O$.

For more details, we show the precision-recall curve for all models as in Figure 11. In both conditions (natural and deceptive gaze), $M D G O$ and $M D G M O$ offer significantly higher area under the curve (auc) value (0.740 and 0.949) compared to $O$ (0.307 and 0.301) 


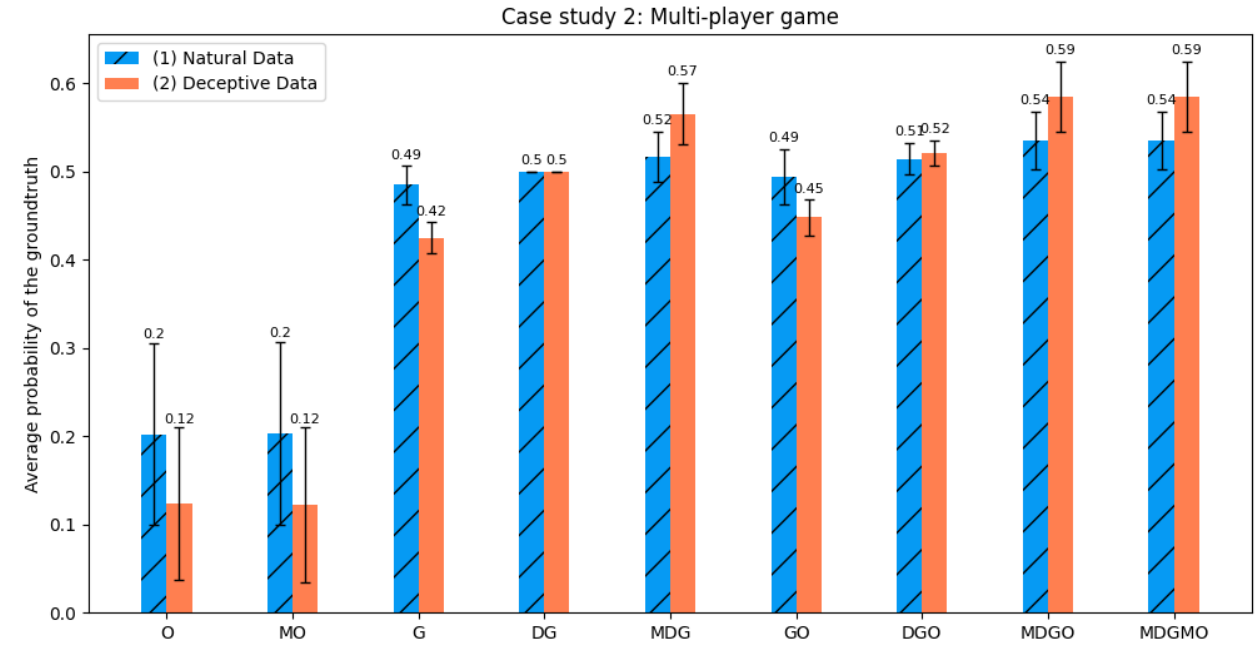

Figure 12: Case study 2: Average ground truth probability of 9 models in 2 conditions: $O=$ Ontic, $M O=$ Modulated Ontic, $G=$ Natural Gaze, DG $=$ Deception Gaze, $M D G=$ Modulated Deception Gaze, GO = Natural Gaze+Ontic, DGO = Deception Gaze+Ontic, $M D G O=$ Modulated Deception Gaze+Ontic, MDGMO = Modulated Deception Gaze + Modulated Ontic. The error bars show 95\% confidence interval.

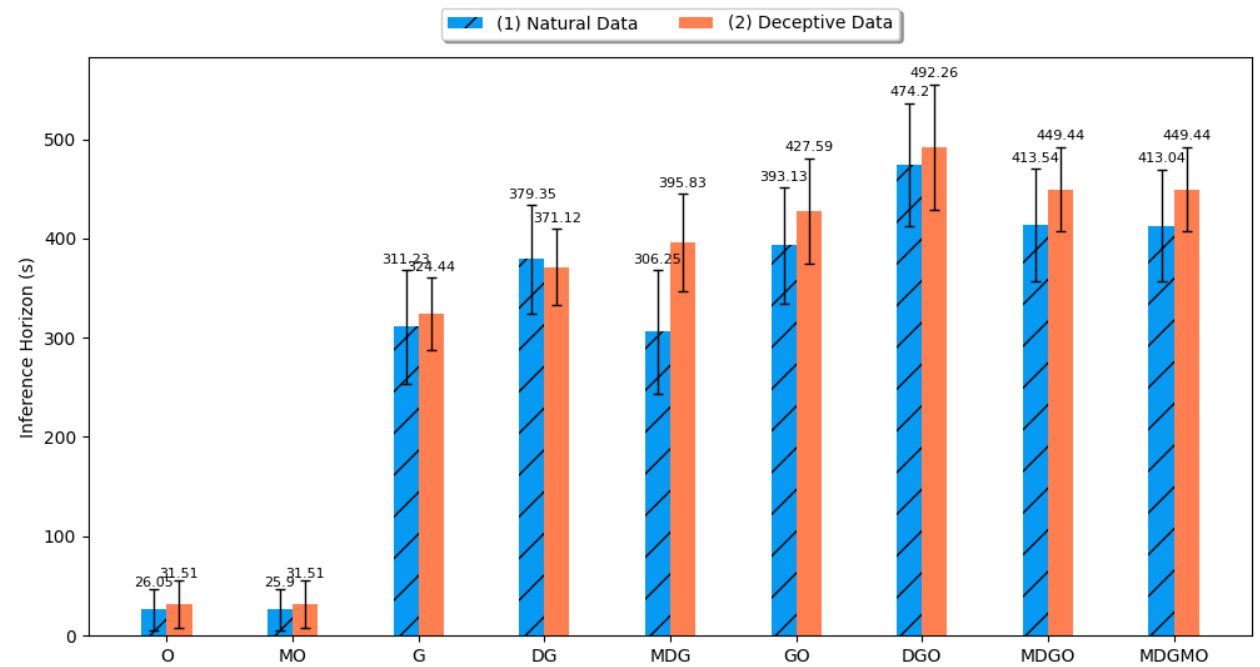

Figure 13: Case study 2: Inference Horizon of 9 models in 2 conditions: $O=$ Ontic, $M O=$ Modulated Ontic, $G=$ Natural Gaze, $D G=$ Deception Gaze, $M D G=$ Modulated Deception Gaze, $G O=$ Natural Gaze+Ontic, DGO = Deception Gaze+Ontic, MDGO = Modulated Deception Gaze+Ontic, MDGMO = Modulated Deception Gaze + Modulated Ontic. The error bars show $95 \%$ confidence interval. 


\begin{tabular}{lrrrrr}
\hline & \multicolumn{2}{c}{ Condition 1 } & & \multicolumn{2}{c}{ Condition 2 } \\
\cline { 2 - 3 } \cline { 6 - 6 } & PRC & Prob & & PRC & Prob \\
\hline $\mathrm{O}$ & 0.307 & 0.2 & & 0.301 & 0.12 \\
$\mathrm{MO}$ & 0.308 & 0.2 & & 0.301 & 0.12 \\
\hline $\mathrm{G}$ & 0.396 & $\mathbf{0 . 4 9}$ & & 0.307 & 0.42 \\
$\mathrm{DG}$ & 0.307 & $\mathbf{0 . 5}$ & & 0.309 & 0.5 \\
$\mathrm{MDG}$ & 0.649 & $\mathbf{0 . 5 2}$ & & 0.921 & 0.57 \\
\hline GO & 0.457 & $\mathbf{0 . 4 9}$ & & 0.308 & 0.45 \\
DGO & 0.721 & $\mathbf{0 . 5 1}$ & & 0.800 & 0.52 \\
MDGO & $\mathbf{0 . 7 4 0}$ & $\mathbf{0 . 5 4}$ & & $\mathbf{0 . 9 4 9}$ & $\mathbf{0 . 5 9}$ \\
MDGMO & $\mathbf{0 . 7 4 0}$ & $\mathbf{0 . 5 4}$ & $\mathbf{0 . 9 4 9}$ & $\mathbf{0 . 5 9}$ \\
\hline
\end{tabular}

Table 2: Accuracy of the Ticket To Ride game: $P R C$ is the area under the curve of the precision-recall curve, Prob is the average ground truth probability. Condition $1=$ Natural gaze; Condition 2 = Deceptive gaze. Winners are highlighted (including the results that are insignificantly different).

and $G O$ ( 0.457 and 0.308$)$. There is also no difference between $O$ and $M O$. When comparing between three Gaze models, $M D G$ performs better than $G$ model. Thus, our proposed models improve the performance in natural and deceptive gaze conditions.

Figure 12 describes the average ground truth probability for nine models in Ticket to Ride game. We applied Wilcoxon signed-rank for the statistical hypothesis test. First, we compare three gaze models: Natural Gaze (G), Deception Gaze (DG) and Modulated Deception Gaze (MDG). Our $M D G$ model offers significantly higher probability than $G$ model on deceptive gaze data $(p<0.05, d=0.99)$. However, there is no difference between $M D G$ and $G$ models on natural data $(p>0.05, d=0.29)$. Considering four Gaze+Ontic models, our proposed model $M D G O$ improves significantly the ground truth probability (59\%) compared to $G O$ model $(45 \%)$ in deceptive condition $(p<0.05, d=1.03)$. In natural condition, there is no significant difference between $M D G O$ model (54\%) and $G O$ model $(49 \%)(p=0.08>0.05, d=0.39)$. In both conditions, there is no difference between $O$ and $M O$; also no difference between $M D G O$ and $M D G M O$.

Considering the inference horizon measures as in Figure 13, there is insignificant difference between $G O$ and $M D G O$ in condition $1(p>0.05)$. However, MDGO offers significant higher inference horizon than $G O$ in condition $2(p<0.05)$. To sum up, our model $M D G O$ performed significantly better in terms of the accuracy when comparing with Natural Gaze+Ontic and Ontic models. MDGO also provides the correct prediction significantly earlier that $O$ model. However, there is no difference in inference horizon between $M D G O$ and $G O$ and also no difference between $M D G M O$ and $M D G O$.

What these results further show is the power of using gaze for intention recognition. In both conditions, the inclusion of gaze data improves both the accuracy and the inference horizon. 


\section{Discussion}

The results show that our new model Modulated Deception Gaze+Ontic (MDGO) is robust on both natural and deceptive data in two case studies. An advantage of our model is that it does not require data collection in a new domain; instead, we simply need a model of the link between intentions and gaze, which is straightforward to define. Moreover, our model provides significantly better accuracy and the inference horizon than the previous state-of-the-art model (Singh et al., 2018, 2020) in both conditions.

In the first case study, we can see that Gaze+Ontic models do not offer higher accuracy than Ontic model. So it is fine to just use the Ontic model in this case. On the other hand, in the second study, Gaze+Ontic models significantly increase the accuracy compared to Ontic model. Therefore, gaze is a useful feature to improve performance in predicting the agents' intentions. The reason for this is because the first study is an easier game, so players might remember the location of the real goal easily without much effort. Thus, they did not have to look at the real goal very often, and as a result the fixation score of the real goal cannot be increased; therefore the probability of this goal cannot be improved when applying the gaze-based model compared to the Ontic model. In contrast, the second study is a complex multi-player game and it requires players to find multiple routes on a map. In this case, remembering the map of North America is a much harder task compared to remembering a single red cell in the first study. Therefore, a player would have to look at the correct routes more often, which expose their real goals.

Moreover, gaze data could be useful at the beginning of the game in the first study. Figure 14 shows the average probability of the ground truth when calculating at a first fifth of the game. Gaze-based models are significantly better than the Ontic model in all three conditions. Although Modulated Deception Gaze+Ontic model does not offer a higher probability than Natural Gaze+Ontic model in the deceptive condition in Figure 14, the main reason is that most players would alter their gaze later when they are more familiar with the task. One thing to note is that gaze data are useful in predicting the real goal earlier in both studies as the inference horizon of gaze-based models is significantly higher than Ontic model. To sum up, gaze data is useful to infer agents' intentions in a complex task requiring them to find their real goals constantly. In a much simpler task, gaze data is useful at the early stage of the task when combining with the planning model. Also, the inclusion of gaze data is valuable for recognising intention earlier in both studies.

One drawback of our model is that it depends on two parameters DistanceThreshold and DurationThreshold. These two measures were selected from the previous work on gaze behaviour (Qvarfordt, 2017). We then evaluate these two parameters on our collected data by making the comparisons between honest and deceptive gaze behaviours in terms of the fixation and distance between the gaze point and the current location of moving action. We find that the natural gaze behaviour in the first study is close to what was proposed in Qvarfordt (2017) (i.e. the average fixation duration of a human is around 300 milliseconds). Given that the domain in Qvarfordt (2017) is a different domain, this result gives empirical support for the use of 300 milliseconds. Also, the fixation count and duration are significantly different between natural and deceptive gaze. A dishonest person would extend fixation duration at the bogus goal and avoid looking at the real one. Furthermore, the distance between the gaze point and the action is significantly greater in deceptive 


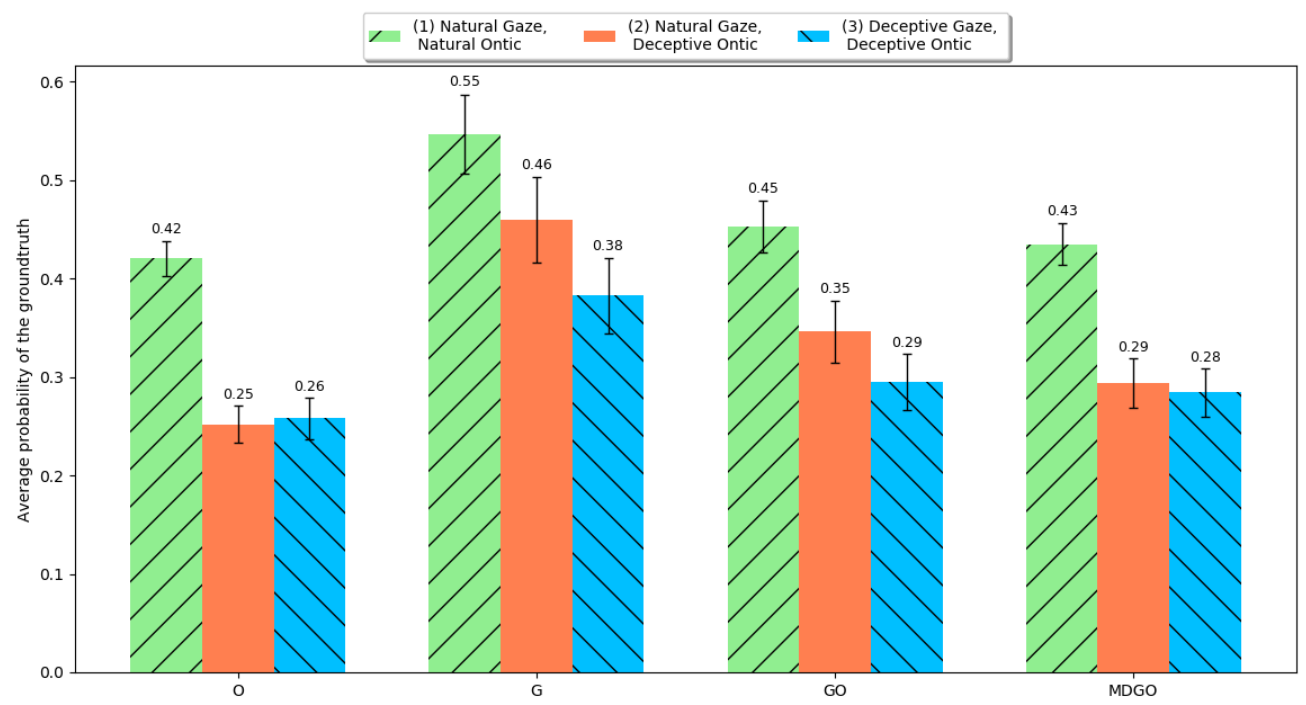

Figure 14: Case study 1: Average ground truth probability of 4 models in 2 conditions at a first fifth of the game: $O=$ Ontic, $G=$ Natural Gaze, $G O=$ Natural Gaze+Ontic, MDGO $=$ Modulated Deception Gaze+Ontic. The error bars show $95 \%$ confidence interval.

behaviour. Based on these results, we argue that the fixation and the distance features are two important features to distinguish between honest and deceptive gaze behaviour.

\section{Conclusion}

In this paper, we present a goal recognition model that uses a combination of human planning and gaze to improve a model-based goal recognition model under deception. Engineers of intelligent systems could use the enhanced models to predict agents' intentions with better accuracy and earlier. This paper is the first investigation that defines probabilistic computational models for deceptive gaze. Our Modulated Deception Gaze+Ontic model achieves promising results and it is a significant improvement to the previous proposed Natural Gaze+ Ontic model in deceptive condition. Moreover, the proposed honesty degree is effective to detect and quantify deception in goal recognition.

In the future, we can apply our models in other domains to further assess the generality of our models. Moreover, the honesty degree was designed by using a sigmoid function with a rate parameter $\alpha$. Currently, there is no approach to find the optimal rate parameter, but we can research more on this to find the suitable rate parameter based on the task. Also, we can examine different threshold value of gaze feature (DistanceThreshold and DurationThreshold) empirically in the honesty degree function to check how the threshold value affects the results.

Finally, goal recognition models that use ontic actions could be extended to handle deceptive actions. Given a more accurate ontic goal recognition model, the accuracy of our combined models should improve too. 
Appendix A. Wilcoxon signed-rank test

\begin{tabular}{|c|c|c|c|}
\hline & Condition 1 & Condition 2 & Condition 3 \\
\hline G vs. DG & $\begin{array}{l}W=7196.0, Z=-10.23 \\
p=1.5 \times 10^{-24}, d=0.59\end{array}$ & $\begin{array}{l}W=19575.0, Z=-2.0 \\
p=0.046, d=0.12\end{array}$ & $\begin{array}{l}\mathrm{W}=16110.0, \mathrm{Z}=-4.3 \\
\mathrm{p}=1.71 \times 10^{-5}, \mathrm{~d}=0.25\end{array}$ \\
\hline G vs. MDG & $\begin{array}{l}W=7413.0, Z=-10.08 \\
p=6.58 \times 10^{-24}, d=0.58\end{array}$ & $\begin{array}{l}W=21783.0, Z=-0.53 \\
p=0.60, d=0.03\end{array}$ & $\begin{array}{l}W=12535.0, Z=-6.68 \\
p=2.44 \times 10^{-11}, d=0.39\end{array}$ \\
\hline DG vs. MDG & $\begin{array}{l}W=18427.5, Z=-2.76 \\
p=0.006, d=0.16\end{array}$ & $\begin{array}{l}\mathrm{W}=17278.0, \mathrm{Z}=-3.52 \\
\mathrm{p}=0.0004, \mathrm{~d}=0.2\end{array}$ & $\begin{array}{l}W=13566.0, Z=-5.99 \\
p=2.08 \times 10^{-9}, d=0.35\end{array}$ \\
\hline GO vs. DGO & $\begin{array}{l}W=20440.0, Z=-1.42 \\
p=0.16, d=0.08\end{array}$ & $\begin{array}{l}W=21921.0, Z=-0.43 \\
p=0.66, d=0.03\end{array}$ & $\begin{array}{l}\mathrm{W}=13372.0, \mathrm{Z}=-6.12 \\
\mathrm{p}=9.36 \times 10^{-10}, \mathrm{~d}=0.35\end{array}$ \\
\hline GO vs. MDGO & $\begin{array}{l}\mathrm{W}=18924.0, \mathrm{Z}=-2.43 \\
\mathrm{p}=0.015, \mathrm{~d}=0.14\end{array}$ & $\begin{array}{l}W=22474.0, Z=-0.07 \\
p=0.95, d=0.0\end{array}$ & $\begin{array}{l}\mathrm{W}=11517.0, \mathrm{Z}=-7.35 \\
\mathrm{p}=1.93 \times 10^{-13}, \mathrm{~d}=0.42\end{array}$ \\
\hline GO vs. MDGMO & $\begin{array}{l}W=15529.0, Z=-4.69 \\
p=2.79 \times 10^{-6}, d=0.27\end{array}$ & $\begin{array}{l}W=21294.0, Z=-0.85 \\
p=0.39, d=0.05\end{array}$ & $\begin{array}{l}\mathrm{W}=14683.0, \mathrm{Z}=-5.25 \\
\mathrm{p}=1.54 \times 10^{-7}, \mathrm{~d}=0.3\end{array}$ \\
\hline DGO vs. MDGO & $\begin{array}{l}W=21285.5, Z=-0.86 \\
p=0.39, d=0.05\end{array}$ & $\begin{array}{l}\mathrm{W}=18763.5, \mathrm{Z}=-2.53 \\
\mathrm{p}=0.01, \mathrm{~d}=0.15\end{array}$ & $\begin{array}{l}W=18744.0, Z=-2.55 \\
p=0.01, d=0.15\end{array}$ \\
\hline DGO vs. MDGMO & $\begin{array}{l}W=11106.5, Z=-7.63 \\
p=2.41 \times 10^{-14}, d=0.44\end{array}$ & $\begin{array}{l}W=19665.5, Z=-1.93 \\
p=0.053, d=0.11\end{array}$ & $\begin{array}{l}\mathrm{W}=14690.0, Z=-5.24 \\
\mathrm{p}=1.58 \times 10^{-7}, \mathrm{~d}=0.3\end{array}$ \\
\hline MDGO vs. MDGMO & $\begin{array}{l}\mathrm{W}=7661.5, \mathrm{Z}=-9.92 \\
\mathrm{p}=3.49 \times 10^{-23}, \mathrm{~d}=0.57\end{array}$ & $\begin{array}{l}\mathrm{W}=16103.5, \mathrm{Z}=-4.3 \\
\mathrm{p}=1.7 \times 10^{-5}, \mathrm{~d}=0.25\end{array}$ & $\begin{array}{l}\mathrm{W}=9483.5, \mathrm{Z}=-8.71 \\
\mathrm{p}=3.15 \times 10^{-18}, \mathrm{~d}=0.5\end{array}$ \\
\hline $\mathrm{O}$ vs. $\mathrm{MO}$ & $\begin{array}{l}W=5318.5, Z=-11.48 \\
p=1.71 \times 10^{-30}, d=0.66\end{array}$ & $\begin{array}{l}\mathrm{W}=16153.5, \mathrm{Z}=-4.27 \\
\mathrm{p}=1.95 \times 10^{-5}, \mathrm{~d}=0.25\end{array}$ & $\begin{array}{l}W=10622.5, Z=-7.95 \\
p=1.88 \times 10^{-15}, d=0.46\end{array}$ \\
\hline O vs. GO & $\begin{array}{l}\mathrm{W}=16374.5, \mathrm{Z}=-4.12 \\
\mathrm{p}=3.73 \times 10^{-5}, \mathrm{~d}=0.24\end{array}$ & $\begin{array}{l}W=19892.5, Z=-1.78 \\
p=0.07, d=0.1\end{array}$ & $\begin{array}{l}W=13992.0, Z=-5.71 \\
p=1.14 \times 10^{-8}, d=0.33\end{array}$ \\
\hline O vs. DGO & $\begin{array}{l}\mathrm{W}=15174.0, \mathrm{Z}=-4.92 \\
\mathrm{p}=8.58 \times 10^{-7}, \mathrm{~d}=0.28\end{array}$ & $\begin{array}{l}\mathrm{W}=18935.0, \mathrm{Z}=-2.42 \\
\mathrm{p}=0.015, \mathrm{~d}=0.14\end{array}$ & $\begin{array}{l}\mathrm{W}=18477.0, \mathrm{Z}=-2.73 \\
\mathrm{p}=0.006, \mathrm{~d}=0.16\end{array}$ \\
\hline $\mathrm{O}$ vs. MDGO & $\begin{array}{l}W=17733.0, Z=-3.22 \\
p=0.001, d=0.19\end{array}$ & $\begin{array}{l}W=17563.0, Z=-3.33 \\
p=0.0009, d=0.19\end{array}$ & $\begin{array}{l}W=22465.0, Z=-0.07 \\
p=0.94, d=0.0\end{array}$ \\
\hline O vs. MDGMO & $\begin{array}{l}W=22341.0, Z=-0.16 \\
p=0.88, d=0.01\end{array}$ & $\begin{array}{l}W=20396.0, Z=-1.45 \\
p=0.15, d=0.08\end{array}$ & $\begin{array}{l}W=17185.0, Z=-3.58 \\
p=0.0003, d=0.21\end{array}$ \\
\hline MO vs. GO & $\begin{array}{l}W=14526.5, Z=-5.35 \\
p=8.68 \times 10^{-8}, d=0.31\end{array}$ & $\begin{array}{l}W=19110.5, Z=-2.3 \\
p=0.02, d=0.13\end{array}$ & $\begin{array}{l}\mathrm{W}=15749.0, \mathrm{Z}=-4.54 \\
\mathrm{p}=5.64 \times 10^{-6}, \mathrm{~d}=0.26\end{array}$ \\
\hline MO vs. DGO & $\begin{array}{l}W=10604.0, Z=-7.96 \\
p=1.71 \times 10^{-15}, d=0.46\end{array}$ & $\begin{array}{l}\mathrm{W}=15442.0, \mathrm{Z}=-4.74 \\
\mathrm{p}=2.1 \times 10^{-6}, \mathrm{~d}=0.27\end{array}$ & $\begin{array}{l}W=17648.0, Z=-3.28 \\
p=0.001, d=0.19\end{array}$ \\
\hline MO vs. MDGO & $\begin{array}{l}W=13556.0, Z=-6.0 \\
p=2.0 \times 10^{-9}, d=0.35\end{array}$ & $\begin{array}{l}\mathrm{W}=15100.0, \mathrm{Z}=-4.97 \\
\mathrm{p}=6.66 \times 10^{-7}, \mathrm{~d}=0.29\end{array}$ & $\begin{array}{l}\mathrm{W}=16114.0, \mathrm{Z}=-4.3 \\
\mathrm{p}=1.73 \times 10^{-5}, \mathrm{~d}=0.25\end{array}$ \\
\hline MO vs. MDGMO & $\begin{array}{l}W=18045.0, Z=-3.01 \\
p=0.0026, d=0.17\end{array}$ & $\begin{array}{l}\mathrm{W}=18833.0, \mathrm{Z}=-2.49 \\
\mathrm{p}=0.013, \mathrm{~d}=0.14\end{array}$ & $\begin{array}{l}W=21124.0, Z=-0.96 \\
p=0.33, d=0.06\end{array}$ \\
\hline
\end{tabular}

Table 3: The Wilcoxon signed-rank test of the average probability of the ground truth in the single-player navigational game. Significant differences $(p<0.05)$ are highlighted. 


\begin{tabular}{|c|c|c|c|}
\hline & Condition 1 & Condition 2 & Condition 3 \\
\hline $\mathrm{O}$ vs. $\mathrm{MO}$ & $\begin{array}{l}W=22575.0, Z=0.0 \\
p=1.0, d=0.0\end{array}$ & $\begin{array}{l}W=22575.0, Z=0.0 \\
p=1.0, d=0.0\end{array}$ & $\begin{array}{l}W=22575.0, Z=0.0 \\
p=1.0, d=0.0\end{array}$ \\
\hline O vs. GO & $\begin{array}{l}\mathrm{W}=16698.5, \mathrm{Z}=-3.96 \\
\mathrm{p}=7.65 \times 10^{-5}, \mathrm{~d}=0.23\end{array}$ & $\begin{array}{l}W=22562.5, Z=-0.01 \\
p=0.99, d=0.0\end{array}$ & $\begin{array}{l}W=11656.0, Z=-7.38 \\
p=1.62 \times 10^{-13}, d=0.43\end{array}$ \\
\hline O vs. MDGO & $\begin{array}{l}\mathrm{W}=18538.5, \mathrm{Z}=-2.72 \\
\mathrm{p}=0.007, \mathrm{~d}=0.16\end{array}$ & $\begin{array}{l}\mathrm{W}=17875.5, \mathrm{Z}=-3.19 \\
\mathrm{p}=0.001, \mathrm{~d}=0.18\end{array}$ & $\begin{array}{l}W=19743.0, Z=-1.95 \\
p=0.052, d=0.11\end{array}$ \\
\hline O vs. MDGMO & $\begin{array}{l}W=22386.5, Z=-0.13 \\
p=0.90, d=0.01\end{array}$ & $\begin{array}{l}\mathrm{W}=17100.0, Z=-3.66 \\
p=0.0003, d=0.21\end{array}$ & $\begin{array}{l}\mathrm{W}=14687.5, \mathrm{Z}=-5.29 \\
\mathrm{p}=1.24 \times 10^{-7}, \mathrm{~d}=0.31\end{array}$ \\
\hline MO vs. GO & $\begin{array}{l}\mathrm{W}=16698.5, \mathrm{Z}=-3.96 \\
\mathrm{p}=7.65 \times 10^{-5}, \mathrm{~d}=0.23\end{array}$ & $\begin{array}{l}W=22562.5, Z=-0.01 \\
p=0.99, d=0.0\end{array}$ & $\begin{array}{l}W=11656.0, Z=-7.38 \\
p=1.62 \times 10^{-13}, d=0.43\end{array}$ \\
\hline MO vs. MDGO & $\begin{array}{l}\mathrm{W}=18538.5, \mathrm{Z}=-2.72 \\
\mathrm{p}=0.007, \mathrm{~d}=0.16\end{array}$ & $\begin{array}{l}\mathrm{W}=17875.5, \mathrm{Z}=-3.19 \\
\mathrm{p}=0.001, \mathrm{~d}=0.18\end{array}$ & $\begin{array}{l}W=19743.0, Z=-1.95 \\
p=0.052, d=0.11\end{array}$ \\
\hline MO vs. MDGMO & $\begin{array}{l}W=22386.5, Z=-0.13 \\
p=0.90, d=0.01\end{array}$ & $\begin{array}{l}W=17100.0, Z=-3.66 \\
p=0.0003, d=0.21\end{array}$ & $\begin{array}{l}\mathrm{W}=14687.5, \mathrm{Z}=-5.29 \\
\mathrm{p}=1.24 \times 10^{-7}, \mathrm{~d}=0.31\end{array}$ \\
\hline GO vs. MDGO & $\begin{array}{l}W=19781.5, Z=-1.88 \\
p=0.06, d=0.11\end{array}$ & $\begin{array}{l}W=19940.5, Z=-1.77 \\
p=0.08, d=0.1\end{array}$ & $\begin{array}{l}\mathrm{W}=11224.5, \mathrm{Z}=-7.62 \\
\mathrm{p}=2.60 \times 10^{-14}, \mathrm{~d}=0.44\end{array}$ \\
\hline GO vs. MDGMO & $\begin{array}{l}\mathrm{W}=16458.5, \mathrm{Z}=-4.11 \\
\mathrm{p}=3.95 \times 10^{-5}, \mathrm{~d}=0.24\end{array}$ & $\begin{array}{l}\mathrm{W}=16661.0, \mathrm{Z}=-3.96 \\
\mathrm{p}=7.63 \times 10^{-5}, \mathrm{~d}=0.23\end{array}$ & $\begin{array}{l}W=18669.5, Z=-2.62 \\
p=0.009, d=0.15\end{array}$ \\
\hline MDGO vs. MDGMO & $\begin{array}{l}\mathrm{W}=18455.5, \mathrm{Z}=-2.96 \\
\mathrm{p}=0.003, \mathrm{~d}=0.17\end{array}$ & $\begin{array}{l}\mathrm{W}=11802.0, Z=-7.34 \\
\mathrm{p}=2.17 \times 10^{-13}, \mathrm{~d}=0.42\end{array}$ & $\begin{array}{l}W=10812.5, Z=-8.04 \\
p=9.18 \times 10^{-16}, d=0.46\end{array}$ \\
\hline
\end{tabular}

Table 4: The Wilcoxon signed-rank test of the inference horizon in the single-player navigational game. Significant differences $(p<0.05)$ are highlighted. 


\begin{tabular}{|c|c|c|}
\hline & Condition 1 & Condition 2 \\
\hline G vs. DG & $W=70.0, Z=-1.27, p=0.20, d=0.29$ & $\mathrm{~W}=0.0, \mathrm{Z}=-4.76, \mathrm{p}=1.91 \times 10^{-6}, \mathrm{~d}=1.07$ \\
\hline G vs. MDG & $W=69.0, Z=-1.31, p=0.19, d=0.29$ & $\mathrm{~W}=3.0, \mathrm{Z}=-4.43, \mathrm{p}=9.54 \times 10^{-6}, \mathrm{~d}=0.99$ \\
\hline DG vs. MDG & $W=57.0, Z=-1.78, p=0.08, d=0.4$ & $\mathrm{~W}=17.0, \mathrm{Z}=-3.54, \mathrm{p}=0.0004, \mathrm{~d}=0.79$ \\
\hline GO vs. DGO & $W=63.0, Z=-1.54, p=0.12, d=0.34$ & $\mathrm{~W}=0.0, \mathrm{Z}=-4.76, \mathrm{p}=1.91 \times 10^{-6}, \mathrm{~d}=1.07$ \\
\hline GO vs. MDGO & $W=58.0, Z=-1.74, p=0.08, d=0.39$ & $\mathrm{~W}=1.0, \mathrm{Z}=-4.62, \mathrm{p}=3.81 \times 10^{-6}, \mathrm{~d}=1.03$ \\
\hline GO vs. MDGMO & $W=59.0, Z=-1.7, p=0.09, d=0.38$ & $\mathrm{~W}=1.0, \mathrm{Z}=-4.62, \mathrm{p}=3.81 \times 10^{-6}, \mathrm{~d}=1.03$ \\
\hline DGO vs. MDGO & $\mathrm{W}=50.0, \mathrm{Z}=-2.05, \mathrm{p}=0.04, \mathrm{~d}=0.46$ & $\mathrm{~W}=18.0, \mathrm{Z}=-3.49, \mathrm{p}=0.0005, \mathrm{~d}=0.78$ \\
\hline DGO vs. MDGMO & $\mathrm{W}=50.0, \mathrm{Z}=-2.05, \mathrm{p}=0.04, \mathrm{~d}=0.46$ & $\mathrm{~W}=18.0, \mathrm{Z}=-3.49, \mathrm{p}=0.0005, \mathrm{~d}=0.78$ \\
\hline MDGO vs. MDGMO & $W=18.0, Z=-0.97, p=0.33, d=0.22$ & $W=6.0, Z=-1.95, p=0.051, d=0.44$ \\
\hline $\mathrm{O}$ vs. MO & $W=10.0, Z=-1.12, p=0.26, d=0.25$ & $W=12.0, Z=-0.34, p=0.74, d=0.08$ \\
\hline $\mathrm{O}$ vs. GO & $\mathrm{W}=16.0, \mathrm{Z}=-3.6, \mathrm{p}=0.0003, \mathrm{~d}=0.8$ & $\mathrm{~W}=10.0, \mathrm{Z}=-3.94, \mathrm{p}=8.2 \times 10^{-5}, \mathrm{~d}=0.88$ \\
\hline O vs. DGO & $\mathrm{W}=12.0, \mathrm{Z}=-3.82, \mathrm{p}=0.0001, \mathrm{~d}=0.85$ & $\mathrm{~W}=5.0, \mathrm{Z}=-4.28, \mathrm{p}=1.91 \times 10^{-5}, \mathrm{~d}=0.96$ \\
\hline O vs. MDGO & $\mathrm{W}=14.0, \mathrm{Z}=-3.71, \mathrm{p}=0.0002, \mathrm{~d}=0.83$ & $\mathrm{~W}=3.0, \mathrm{Z}=-4.43, \mathrm{p}=9.54 \times 10^{-6}, \mathrm{~d}=0.99$ \\
\hline O vs. MDGMO & $\mathrm{W}=15.0, \mathrm{Z}=-3.65, \mathrm{p}=0.0003, \mathrm{~d}=0.82$ & $\mathrm{~W}=3.0, \mathrm{Z}=-4.43, \mathrm{p}=9.54 \times 10^{-6}, \mathrm{~d}=0.99$ \\
\hline MO vs. GO & $\mathrm{W}=16.0, \mathrm{Z}=-3.6, \mathrm{p}=0.0003, \mathrm{~d}=0.8$ & $\mathrm{~W}=8.0, \mathrm{Z}=-4.07, \mathrm{p}=4.77 \times 10^{-5}, \mathrm{~d}=0.91$ \\
\hline MO vs. DGO & $\mathrm{W}=12.0, \mathrm{Z}=-3.82, \mathrm{p}=0.0001, \mathrm{~d}=0.85$ & $\mathrm{~W}=5.0, \mathrm{Z}=-4.28, \mathrm{p}=1.91 \times 10^{-5}, \mathrm{~d}=0.96$ \\
\hline MO vs. MDGO & $\mathrm{W}=14.0, \mathrm{Z}=-3.71, \mathrm{p}=0.0002, \mathrm{~d}=0.83$ & $\mathrm{~W}=5.0, \mathrm{Z}=-4.28, \mathrm{p}=1.91 \times 10^{-5}, \mathrm{~d}=0.96$ \\
\hline MO vs. MDGMO & $\mathrm{W}=14.0, \mathrm{Z}=-3.71, \mathrm{p}=0.0002, \mathrm{~d}=0.83$ & $\mathrm{~W}=5.0, \mathrm{Z}=-4.28, \mathrm{p}=1.91 \times 10^{-5}, \mathrm{~d}=0.96$ \\
\hline
\end{tabular}

Table 5: The Wilcoxon signed-rank test of the average probability of the ground truth in the multi-player game. Significant differences $(p<0.05)$ are highlighted.

\begin{tabular}{|c|c|c|}
\hline & Condition 1 & Condition 2 \\
\hline $\mathrm{O}$ vs. MO & $W=10.0, Z=-1.12, p=0.26, d=0.25$ & $W=12.0, Z=-0.34, p=0.74, d=0.08$ \\
\hline O vs. GO & $\mathrm{W}=16.0, \mathrm{Z}=-3.6, \mathrm{p}=0.0003, \mathrm{~d}=0.8$ & $\mathrm{~W}=10.0, \mathrm{Z}=-3.94, \mathrm{p}=8.20 \times 10^{-5}, \mathrm{~d}=0.88$ \\
\hline O vs. MDGO & $\mathrm{W}=14.0, \mathrm{Z}=-3.71, \mathrm{p}=0.0002, \mathrm{~d}=0.83$ & $\mathrm{~W}=3.0, \mathrm{Z}=-4.43, \mathrm{p}=9.54 \times 10^{-6}, \mathrm{~d}=0.99$ \\
\hline $\mathrm{O}$ vs. MDGMO & $\mathrm{W}=15.0, \mathrm{Z}=-3.65, \mathrm{p}=0.0003, \mathrm{~d}=0.82$ & $\mathrm{~W}=3.0, \mathrm{Z}=-4.43, \mathrm{p}=9.54 \times 10^{-6}, \mathrm{~d}=0.99$ \\
\hline MO vs. GO & $\mathrm{W}=16.0, \mathrm{Z}=-3.6, \mathrm{p}=0.0003, \mathrm{~d}=0.8$ & $\mathrm{~W}=8.0, \mathrm{Z}=-4.07, \mathrm{p}=4.77 \times 10^{-5}, \mathrm{~d}=0.91$ \\
\hline MO vs. MDGO & $\mathrm{W}=14.0, \mathrm{Z}=-3.71, \mathrm{p}=0.0002, \mathrm{~d}=0.83$ & $\mathrm{~W}=5.0, \mathrm{Z}=-4.28, \mathrm{p}=1.91 \times 10^{-5}, \mathrm{~d}=0.96$ \\
\hline MO vs. MDGMO & $\mathrm{W}=14.0, \mathrm{Z}=-3.71, \mathrm{p}=0.0002, \mathrm{~d}=0.83$ & $\mathrm{~W}=5.0, \mathrm{Z}=-4.28, \mathrm{p}=1.91 \times 10^{-5}, \mathrm{~d}=0.96$ \\
\hline GO vs. MDGO & $W=58.0, Z=-1.74, p=0.08, d=0.39$ & $\mathrm{~W}=1.0, \mathrm{Z}=-4.62, \mathrm{p}=3.81 \times 10^{-6}, \mathrm{~d}=1.03$ \\
\hline GO vs. MDGMO & $W=59.0, Z=-1.7, p=0.09, d=0.38$ & $\mathrm{~W}=1.0, \mathrm{Z}=-4.62, \mathrm{p}=3.81 \times 10^{-6}, \mathrm{~d}=1.03$ \\
\hline MDGO vs. MDGMO & $W=18.0, Z=-0.97, p=0.33, d=0.22$ & $W=6.0, Z=-1.95, p=0.051, d=0.44$ \\
\hline
\end{tabular}

Table 6: The Wilcoxon signed-rank test of the inference horizon in the multi-player game. Significant differences $(p<0.05)$ are highlighted. 


\section{References}

Admoni, H., \& Srinivasa, S. (2016). Predicting user intent through eye gaze for shared autonomy. In 2016 AAAI Fall Symposium Series.

Aravena, C., Vo, M., Gao, T., Shiratori, T., Yu, L.-F., \& Contributors, E. (2017). Perception meets examination: Studying deceptive behaviors in vr.. In CogSci.

Bednarik, R., Eivazi, S., \& Vrzakova, H. (2013). A computational approach for prediction of problem-solving behavior using support vector machines and eye-tracking data. In Eye Gaze in Intelligent User Interfaces, pp. 111-134. Springer.

Bell, J. B. (2003). Toward a theory of deception. International journal of intelligence and counterintelligence, 16(2), 244-279.

Bhaskaran, N., Nwogu, I., Frank, M. G., \& Govindaraju, V. (2011). Lie to me: Deceit detection via online behavioral learning. In Face and Gesture 2011, pp. 24-29.

Carberry, S. (2001). Techniques for plan recognition. User Modeling and User-Adapted Interaction, 11(1-2), 31-48.

Demyanov, S., Bailey, J., Ramamohanarao, K., \& Leckie, C. (2015). Detection of Deception in the Mafia Party Game. In Proceedings of the 2015 ACM on International Conference on Multimodal Interaction, pp. 335-342.

Duchowski, A. T. (2007). Eye tracking methodology. Theory and practice, 328(614), 2-3.

Duchowski, A. T. (2018). Gaze-based interaction: A 30 year retrospective. Computers \& Graphics, 73, 59-69.

Gal, Y., Reddy, S., Shieber, S. M., Rubin, A., \& Grosz, B. J. (2012). Plan recognition in exploratory domains. Artificial Intelligence, 176(1), 2270 - 2290.

Geib, C., \& Goldman, R. (2001). Plan recognition in intrusion detection systems.. Vol. 1, pp. $46-55$ vol.1.

Hausegger, T., Vater, C., \& Hossner, E.-J. (2019). Peripheral vision in martial arts experts: The cost-dependent anchoring of gaze. Journal of Sport and Exercise Psychology, 41(3), 137-145.

Huang, C.-M., Andrist, S., Sauppé, A., \& Mutlu, B. (2015). Using gaze patterns to predict task intent in collaboration. Frontiers in psychology, 6, 1049.

Keren, S., Gal, A., \& Karpas, E. (2015). Goal recognition design for non-optimal agents. In Twenty-Ninth AAAI Conference on Artificial Intelligence.

Koochaki, F., \& Najafizadeh, L. (2019). Eye gaze-based early intent prediction utilizing cnn-lstm. In 2019 41st Annual International Conference of the IEEE Engineering in Medicine and Biology Society (EMBC), pp. 1310-1313. IEEE.

Land, M. F. (2009). Vision, eye movements, and natural behavior. Visual neuroscience, $26(1), 51-62$.

Mann, S., Vrij, A., Leal, S., Granhag, P. A., Warmelink, L., \& Forrester, D. (2012). Windows to the Soul? Deliberate Eye Contact as a Cue to Deceit. Journal of Nonverbal Behavior, 36(3), 205-215. 
Masters, P., \& Sardina, S. (2017a). Cost-based goal recognition for path-planning. In Proceedings of the 16th Conference on Autonomous Agents and MultiAgent Systems, pp. $750-758$.

Masters, P., \& Sardina, S. (2017b). Deceptive path-planning.. In IJCAI, pp. 4368-4375.

Masters, P., \& Sardina, S. (2019). Goal recognition for rational and irrational agents. In Proceedings of the 18th International Conference on Autonomous Agents and MultiAgent Systems, pp. 440-448. International Foundation for Autonomous Agents and Multiagent Systems.

Newn, J., Allison, F., Velloso, E., \& Vetere, F. (2018). Looks can be deceiving: Using gaze visualisation to predict and mislead opponents in strategic gameplay. In Proceedings of the 2018 CHI Conference on Human Factors in Computing Systems, pp. 1-12.

Newn, J., Velloso, E., Allison, F., Abdelrahman, Y., \& Vetere, F. (2017). Evaluating realtime gaze representations to infer intentions in competitive turn-based strategy games. In Proceedings of the Annual Symposium on Computer-Human Interaction in Play, CHI PLAY '17, pp. 541-552, New York, NY, USA. Association for Computing Machinery.

Pereira, R. F., Oren, N., \& Meneguzzi, F. (2017). Landmark-based heuristics for goal recognition. In Thirty-First AAAI Conference on Artificial Intelligence.

Pérez-Rosas, V., Abouelenien, M., Mihalcea, R., \& Burzo, M. (2015). Deception detection using real-life trial data. In Proceedings of the 2015 ACM on International Conference on Multimodal Interaction, ICMI '15, p. 59-66, New York, NY, USA. Association for Computing Machinery.

Proudfoot, J. G., Jenkins, J. L., Burgoon, J. K., \& Nunamaker Jr, J. F. (2016). More than meets the eye: How oculometric behaviors evolve over the course of automated deception detection interactions. Journal of Management Information Systems, 33(2), $332-360$.

Qvarfordt, P. (2017). Gaze-informed multimodal interaction. In The Handbook of Multimodal-Multisensor Interfaces: Foundations, User Modeling, and Common Modality Combinations-Volume 1, pp. 365-402. Association for Computing Machinery and Morgan \& Claypool.

Ramírez, M., \& Geffner, H. (2010). Probabilistic plan recognition using off-the-shelf classical planners. In Twenty-Fourth AAAI Conference on Artificial Intelligence.

Ramírez, M., \& Geffner, H. (2009). Plan recognition as planning. In Twenty-First International Joint Conference on Artificial Intelligence.

Singh, R., Miller, T., Newn, J., Sonenberg, L., Velloso, E., \& Vetere, F. (2018). Combining planning with gaze for online human intention recognition. In Proceedings of the 17th International Conference on Autonomous Agents and MultiAgent Systems, pp. 488-496. International Foundation for Autonomous Agents and Multiagent Systems.

Singh, R., Miller, T., Newn, J., Velloso, E., Vetere, F., \& Sonenberg, L. (2020). Combining gaze and ai planning for online human intention recognition. Artificial Intelligence, 284, 103275. 
Sukthankar, G., Geib, C., Bui, H. H., Pynadath, D., \& Goldman, R. P. (2014). Plan, activity, and intent recognition: Theory and practice. Newnes.

Vered, M., Kaminka, G. A., \& Biham, S. (2016). Online goal recognition through mirroring: Humans and agents. In The Fourth Annual Conference on Advances in Cognitive Systems.

Whaley, B. (1982). Toward a general theory of deception. The Journal of Strategic Studies, $5(1), 178-192$.

Wheeler, M. (2004). True or false? lie detection at the bargaining table. Negotiation, 11 .

Wood, G., Vine, S. J., Parr, J., \& Wilson, M. R. (2017). Aiming to deceive: Examining the role of the quiet eye during deceptive aiming actions. Journal of Sport and Exercise Psychology, 39(5), 327-338.

Zhou, L., \& Zhang, D. (2012). Automatic deception detection in computer-mediated communication. IEEE Intelligent Systems, 27, 73-75. 